\title{
Flutuações do choque no processo de Hammersley
}

\author{
Marcio Watanabe Alves de Souza
}

\author{
TESE APRESENTADA AO \\ Instituto de Matemática E Estatística da \\ Universidade de SÃo Paulo \\ PARA OBTENÇÃO DO TÍTULO DE \\ DOUTOR EM CIÊNCIAS
}

\author{
Programa: Estatística
}

Orientador: Prof. Dr. Leandro Pinto Rodrigues Pimentel

Durante o desenvolvimento deste trabalho o autor recebeu auxílio financeiro do CNPQ 


\section{Flutuações do choque no processo de Hammersley}

Este exemplar corresponde à redação

final da tese devidamente corrigida

e defendida por Marcio Watanabe Alves de Souza

e aprovada pela Comissão Julgadora.

Banca Examinadora:

- Prof. Dr. Leandro P. Rodrigues Pimentel (orientador) - UFRJ

- Prof. Dr. Cristian F. Coletti - UFABC

- Prof. Dr. Fábio Prates Machado - USP

- Prof. Dr. Luiz Renato Gonçalves Fontes - USP

- Prof. Dr. Serguei Popov - UNICAMP 
Matemática rimaria perfeitamente com fantástica se assim me ajudasse a sorvê-la, mas a própria palavra fantástica que por ser tão performática e já ter tantas derivadas, se somada à matemática me exauriria para resolvê-las.

Porém, ao integrá-las me excitaria: somaria suas áreas com partiçôes cada vez mais ralas. Começaria na reta de maneira clara, até o ápice do deslumbre, adicionando mais duas dimensões atingiria o volume. Usaria os óculos da física que por ser mais empirista me daria grande emoção quando da inércia me arrebataria, como por definição e com total abstração depois de sair da lama do chão tenderia ao infinito onde tudo é mais bonito.

Neste espaço perfeito, que não pertence só aos eleitos, tem verdade, tem magia, sangue, suor e alegria.

Topologicamente falando o universo cabe no cartesiano.

Refutando todo engano provarei por absurdo que este universo cabe no mundo e todos eles no plano.

Seja a Matemática algo sobre-humano há uma função fantástica convergindo para zero: toda rima e sua inversa também é obra prima. 
por desmistificar essa essência redefinindo limites e acreditar que em mim também cabe esta consciência.

Fernanda Di Genio Watanabe 


\section{Agradecimentos}

Obrigado Deus. Obrigado ao Leandro pela orientação. Obrigado ao meu pai e à minha mãe. Obrigado à minha esposa Fernanda e aos meus filhos Tetsuo e Suezo por tudo, em especial pela paciência, vocês são minha vida. 


\section{Resumo}

No presente trabalho provamos resultados sobre as flutuações dos fluxos de partículas e das partículas marcadas no processo de Hammersley multiclasse. Os métodos das demonstrações são robustos, formulados de modo a serem aplicados em outros processos, em particular se aplicam ao processo de exclusão totalmente assimétrico multiclasse (TASEP multiclasse) e à seu respectivo modelo de percolação de última passagem. Os principais teoremas obtidos são um teorema central do limite para o choque, seu coeficiente de difusão e uma fórmula exata para a variância do fluxo de partículas de classe $N \geq 2$ para o processo em equilíbrio multiclasse.

Palavras-chave: Processo de Hammersley, Percolação de última passagem, Processo de exclusão totalmente assimétrico, Processo multiclasse, Flutuações do choque. 


\section{Abstract}

We prove fluctuations results concerning fluxes of particles and tagged particles on multiclass Hammersley process. The methods used are robust and apply to other processes, in particular all the proofs can be adapted to the Multiclass totally asymmetric simple exclusion process (Multiclass TASEP) and its respective last passage percolation model. The main theorems obtained are a central limit theorem for the shock, its diffusion coefficient and an exact formula for the variance of the $N$-th class particle flux in a stationary version of the multiclass process when $N \geq 2$.

Keywords: Hammersley process, Last passage percolation, Totally asymmetric exclusion process, Multiclass process, Shock fluctuations. 


\section{Sumário}

1 Introdução 1

Organização da Tese . . . . . . . . . . . . . . . . . . . . 4

2 Modelos e Resultados 5

Processo de Hammersley . . . . . . . . . . . . . . . . . . . . . . 5

Processo de Hammersley em uma caixa finita . . . . . . . . . . . . 8

Choque e resultados . . . . . . . . . . . . . . . . . . . . 8

Processo multiclasse . . . . . . . . . . . . . . . . . . 14

3 Preliminares $\quad 16$

Choque e processo de duas classes no equilíbrio . . . . . . . . . . . 16

Fluxo de partículas de segunda classe no equilíbrio . . . . . . . . . . 21

Medida invariante multiclasse . . . . . . . . . . . . . . . . . 22

4 Teorema Central do Limite 25

5 Coeficiente de difusão 33

6 Variância do Fluxo no Equilíbrio 49

Referências Bibliográficas $\quad 63$ 


\section{Capítulo 1}

\section{Introdução}

O Processo de Hammersley é um sistema de partículas em $\mathbb{R}$ em que cada partícula salta para uma posição uniformemente escolhida entre a sua posição e a posição da partícula mais próxima a sua esquerda. A taxa de salto é uma variável exponencial de parâmetro proporcional ao espaço que a partícula dispõe para saltar. Podemos descrevê-lo de modo mais preciso através de sua construção gráfica. Seja $\nu$ a configuração inicial, aleatória ou não, do processo e seja $P$ um processo de Poisson homogêneo em $\mathbb{R}^{2}$. Fazemos uma realização do processo deslocando o eixo inicial pela coordenada do tempo e cada vez que uma marca de Poisson é intersectada a partícula mais próxima à direita salta para esta marca. Nesta construção, cada realização pode ser vista como um conjunto de trajetórias no espaço-tempo $\mathbb{R}^{2}$ que não se cruzam. A versão descrita acima é devida a Aldous e Diaconis e por este motivo o processo também é conhecido como processo HAD (Hammersley-Aldous-Diaconis). Os processos de Poisson de taxa $\lambda>0$ são invariantes para essa dinâmica [22].

A primeira versão do processo de Hammersley foi criada junto com seu modelo de percolação de última passagem em [20], onde Hammersley propôs uma solução para o problema de Ulam, que estuda o comportamento limite da maior subsequência crescente $L_{n}$ de uma permutação aleatória do conjunto $\{1,2, \ldots, n\}$. Baik e Rains [2], Cator e Groeneboom [8] [9] e Groeneboom [19] estudaram a versão do processo restrita a uma caixa finita com bordo.

Uma das variáveis de maior interesse em sistemas de partículas conservativos é o fluxo ou corrente de partículas visto por um observador com velocidade $V_{t}$. O fluxo é definido como o número de partículas que ultrapassam o observador da direita para a esquerda menos o número de partículas que ultrapassam o observador da esquerda para a direita no intervalo de tempo $(0, t]$. Em [15], Ferrari e Fontes provaram que a variância do fluxo ao longo da característica é sub-linear para o processo de exclusão assimétrico (ASEP). Bálazs [4] generalizou esse re- 
sultado para uma classe de modelos de deposição. Em [21] e [3], Johansson, Baik e Deift deram as primeiras demonstrações rigorosas de que as flutuações do fluxo em processos de percolação de última passagem (Hammersley e TASEP) são da ordem de $t^{1 / 3}$. Desde então, muita atenção tem sido dada para estender esses resultados de flutuações no regime estacionário a outros processos (ver [18], [9], [2], [6] entre outros).

O comportamento hidrodinâmico macroscópico do processo de Hammersley é dado pela equação de Burgers $\partial_{t} u+\partial_{x} g(u)=0$, onde $g(u)=\frac{1}{u}$ [22]. Quando as condições iniciais são $u(r, 0)=\lambda$ para $r>0$ e $u(r, 0)=\frac{1}{\rho}$ quando $r \leq 0$, a solução tem uma mudança drástica quando o produto $\lambda \rho$ varia de valores menores que 1 para valores maiores que 1 . Para $\lambda \rho=1$, o sistema é estacionário e as soluções são constantes no tempo. Para $\lambda \rho<1$ o sistema desenvolve um fronte de rarefação enquanto para $\lambda \rho>1$, o sistema desenvolve um choque macroscópico. No processo de Hammersley, Baik e Rains [2] provaram um teorema central do limite para o fluxo nos três regimes. Para o processo de exclusão (TASEP), Ben Arous e Corwin [7] compilaram os resultados de [18], [14] e [5] que provam o teorema central do limite para o fluxo no equilíbrio, no choque e no fronte de rarefação, respectivamente. No presente trabalho, nosso foco principal será estudar as flutuações em sistemas no regime de choque.

A estrutura microscópica do choque pode ser descrita pela partícula de segunda classe [13]. A partícula de segunda classe $Z_{t}$ é uma partícula que possui uma movimentação distinta das outras partículas, as quais chamaremos daqui em diante de partículas de primeira classe. Ao invés de saltar sobre as marcas de salto $\mathbf{P}$, a partícula de segunda classe salta para frente, para a posição onde se encontrava uma partícula de primeira classe que acabou de ultrapassá-la. A importância das partículas de segunda classe é que elas são o análogo microscópico das características das equações diferenciais de Burgers que descrevem o comportamento ao longo do tempo de perturbações da medida inicial.

Ferrari e Fontes [14] são a principal referência no estudo de flutuações do choque microscópico. Eles provaram um teorema da condição inicial para $Z_{t}$ no processo de exclusão assímetrico, obtendo entre outros resultados o coeficiente de difusão e o teorema central do limite para $Z_{t}$. Para o processo de Hammersley, Seppäläinen [23] provou um teorema central do limite para o fluxo no regime de choque gerado por uma classe grande de medidas iniciais.

Nós utilizamos os recentes avanços sobre o fluxo no equilíbrio para provar um teorema central do limite para o choque $Z_{t}$ no processo de Hammersley e calculamos o coeficiente de difusão $D:=\lim _{\infty} \frac{\operatorname{Var} Z_{t}}{t}$. De fato, mostramos que a 
distância entre $t Z_{t}$ e $\operatorname{Var} Z_{t}$ vai a zero na escala $t^{\frac{2}{3}+\epsilon}$, para todo $\epsilon>0$. Prosseguimos mostrando uma fórmula para a variância do fluxo de partículas de segunda classe para o processo multiclasse em equilíbrio. Utilizamos a representação da medida invariante como a saída de uma fila $M / M / 1$ [16].

Partículas de segunda classe podem ser representadas pelas diferenças entre dois processos acoplados, gerando um processo com duas classes de partículas [14] [9]. Do mesmo modo, podemos acoplar $n$ processos e obter um processo multiclasse. Ao contrário do que acontece no processo com uma classe de partículas, o conjunto das medidas invariantes para o processo multiclasse não é Poisson para cada classe de partícula. Ferrari e Martin [16] [17] provaram que as únicas medidas invariantes extremas são obtidas como a saída de um sistema de $n-1$ filas em série com n classes de clientes. A descrição do sistema é a seguinte: Clientes de primeira classe chegam à primeira fila como um processo de Poisson de taxa $\rho_{1}$. Para cada fila $k$ com $k$ de 1 a n-1, existe uma quantidade infinita de clientes de classe $k+1$ esperando por atendimento. Quando um cliente de classe i é atendido na fila $k$, ele passa para a próxima fila ou sai do sistema caso $k=n-1$. O atendimento segue a regra FIFO para clientes de mesma classe, ou seja, o primeiro a chegar é o primeiro a sair. Clientes de classe i têm prioridade no atendimento com relação a clientes de classes maiores. Assim, por exemplo, quando um cliente de segunda classe chega na segunda fila, ele é atendido antes dos clientes de terceira classe que lá estavam. Por fim, as taxas de serviço do servidor da fila $k$ são exponenciais independentes de parâmetros $\rho_{k}$. Cator e Pimentel [10] recentemente usaram funções de Busemman para obter a medida invariante multiclasse para uma generalização do processo de Hammersley.

Terminamos essa introdução com um comentário sobre as provas dos resultados. Apesar de estarem escritas para o processo de Hammersley, nossa intenção era utilizar métodos robustos o suficiente para que as mesmas provas, com poucas adaptações, pudessem ser utilizadas em outros processos. Em especial, todos os resultados do texto podem ser adaptados para o processo de exclusão totalmente assimétrico e seu respectivo modelo de percolação de última passagem. 


\section{Organização da Tese}

Este trabalho está estruturado da seguinte forma:

No capítulo 2 definimos os principais modelos, variáveis e resultados. Começamos com as definiões do processo de Hammersley em $\mathbb{R}$ e numa caixa finita, além de seu respectivo modelo de percolação de última passagem. Definimos fluxo de partículas de primeira e segunda classes e partículas marcadas. Damos a definição formal de choque e enunciamos os principais teoremas. Terminamos o capítulo definindo o processo de Hammersley multiclasse.

O capítulo 3 traz algumas propriedades básicas utilizadas ao longo do texto. Mostramos a relação entre o choque e a posição de uma partícula de segunda classe no processo de duas classes. Descrevemos a medida invariante multiclasse como a saída de uma fila com várias classes de clientes e mostramos que, para o processo em equilíbrio multiclasse, os fluxos verticais também podem ser vistos como a saída de filas com várias classes de clientes.

No capítulo 4 mostramos um teorema sobre a influência da condição inicial nas flutuações do fluxo. Como corolário, obtemos teoremas centrais do limite para o fluxo, para a partícula marcada e para o choque microscópico no processo de Hammersley.

O capítulo 5 é dedicado ao cálculo da variância do choque. Mostramos como relacionar os momentos do fluxo com a posição da partícula marcada e como esse método se aplica no caso do choque. À partir dessa relação, cálculamos a esperança para todo tempo $t$ e o coeficiente de difusão do choque.

Encerramos, no capítulo 6, com uma fórmula exata para a variância do fluxo de partículas de classe $n \geq 2$ para o processo em equilíbrio multiclasse. Em [9], Cator e Groeneboom demonstram uma fórmula análoga para fluxo de partículas de primeira classe e a utilizam para mostrar que a ordem das flutuações do fluxo ao longo da direção característica pertence a classe de KPZ $t^{1 / 3}$. 


\section{Capítulo 2}

\section{Modelos e Resultados}

Neste capítulo damos as definições formais dos modelos e das principais variáveis aleatórias estudadas no restante da tese. Também enunciamos os principais resultados.

\section{Processo de Hammersley}

Começamos com a definição do Processo de Hammersley. Nós usaremos uma construção gráfica a la Harris (ver [1] para mais detalhes).

Para cada medida $\nu$ em $\mathbb{R}$ associamos um processo não decrescente $\nu(\cdot)$ definido por

$$
\nu(x)= \begin{cases}\nu((0, x]) & \text { para } x \geq 0 \\ -\nu((x, 0]) & \text { para } x<0\end{cases}
$$

Denotamos por $\mathcal{X}$ o espaço de estados. Aqui escolhemos $\mathcal{X}$ como sendo o conjunto de todas as medidas positivas localmente finitas $\nu$ tal que

$$
\liminf _{y \rightarrow-\infty} \frac{\nu(y)}{y}>0
$$

Esta condição evita que as partículas saltem instantaneamente para menos infinito. Denotamos o processo de Hammersley com medida inicial $\nu$ por $\left(M_{t}^{\nu}\right)_{t \in \mathbb{R}}$, onde $M_{t}^{\nu} \in \mathcal{X}$ para todo tempo t. Os pontos de $M_{t}^{\nu}$ são chamados de partículas e a dinâmica dos saltos dessas partículas ao longo do tempo é determinada pelo processo de Poisson $\mathbf{P}$ com intensidade $1 \mathrm{em} \mathbb{R}^{2}$ do seguinte modo: se $\left(x_{0}, t\right) \in \mathbf{P}$ então $M_{t}^{\nu}\left(\left\{x_{0}\right\}\right)=1$ e para $x<x_{0}$ temos $M_{t}^{\nu}(\{x\})=M_{t-}^{\nu}(\{x\})$. Já para $x>x_{0}$ temos $M_{t}^{\nu}\left(\left(x_{0}, x\right]\right)=\left(M_{t-}^{\nu}\left(\left(x_{0}, x\right]\right)-1\right) \mathbf{1}_{\left[M_{t-}^{\nu}\left(\left(x_{0}, x\right]\right)-1 \geq 0\right]}$. Fixando a configuração inicial $\nu_{0}=\nu$ e o processo de saltos $\mathbf{P}=\omega$, temos que o processo 
$\left(M_{t}^{\nu}\right)_{t \in \mathbb{R}}$ é uma função determinística $\psi$ de $\nu$ e $\omega$, isto é, $M_{t}^{\nu}=\psi(t, \nu, \omega)$.

Os processos de Poisson em $\mathbb{R}$ constituem a única classe de medidas invariantes ergódicas para o processo de Hammersley. Denotamos por $\nu_{\lambda}$ um processo de Poisson de taxa $\lambda \geq 0$.

Uma da principais variáveis de interesse em sistemas de partículas é o fluxo ou corrente de partículas que passam por um observador que viaja a uma certa velocidade $V_{t}$. Definimos o fluxo entre dois pontos de $\mathbb{R} \times \mathbb{R}_{+}$num processo de Hammersley com medida inicial $\nu$ a seguir.

Sejam $x, y \in \mathbb{R}$ e $s, t \geq 0$. Considere $t \geq s$. Definimos o fluxo positivo $L_{\nu}((y, s),(x, t))_{+}$de $y$ a $x$ no tempo $t$ como sendo o total de partículas que estão à direita de $y$ no tempo $s$ e à esquerda de $x$ (inclusive) no tempo $t$. Do mesmo modo, definimos o fluxo negativo $L_{\nu}((y, s),(x, t))_{-}$de $y$ a $x$ no tempo $t$ como sendo o total de partículas que estão à esquerda de $y$ (inclusive) no tempo $s$ e à direita de $x$ no tempo $t$. Definimos o fluxo $L_{\nu}$ como

$$
L_{\nu}((y, s),(x, t)):=L_{\nu}((y, s),(x, t))_{+}-L_{\nu}((y, s),(x, t))_{-}
$$

Por fim, denotamos o fluxo da origem a $x$ no tempo $t$ por

$$
L_{\nu}(x, t):=L_{\nu}((0,0),(x, t))_{+}-L_{\nu}((0,0),(x, t))_{-}
$$

Para o caso $t<s$, definimos o fluxo por anti-simetria, ie, para todo $x, y \in \mathbb{R}$ e $t<s, L_{\nu}((y, s),(x, t)):=-L_{\nu}((x, t),(y, s))$. Quando a medida inicial for a medida invariante $\nu_{\lambda}$ Poisson de taxa $\lambda$, para simplificar a notação, escreveremos $L_{\lambda}$ no lugar de $L_{\nu_{\lambda}}$.

Outra importante propriedade do fluxo que utilizaremos nas provas, além da (i) anti-simetria, é a (ii) aditividade, ie, $\forall x, y, z \in \mathbb{R}$ e $r, s, t \geq 0$, temos que:

$$
\begin{gathered}
(i) \quad L_{\nu}((x, s),(y, t))=-L_{\nu}((y, t),(x, s)) \\
(\text { ii }) \quad L_{\nu}((x, s),(y, t))=L_{\nu}((x, s),(z, r))+L_{\nu}((z, r),(y, t))
\end{gathered}
$$

Também temos interesse em estudar a trajetória de uma partícula fixada. 
Considere o processo de Hammersley com medida inicial invariante $\nu_{\lambda}$. Acrescentamos uma partícula na origem do processo no tempo zero. Definimos a partícula marcada $X(t)$ como sendo a posição no tempo t da partícula cuja posição inicial é a origem. Escolhemos essa partícula, pois ela representa uma partícula típica para a medida $\nu_{\lambda}$, já que tanto o processo a sua direita quanto o processo a sua esquerda são Poisson de taxa $\lambda$.

O processo de Hammersley pode ser visto como um modelo de percolação de última passagem [1] que definimos abaixo.

Novamente $\mathbf{P} \subseteq \mathbb{R}^{2}$ denota um processo de Poisson bidimensional de intensidade 1 .

Para cada $\mathbf{p}, \mathbf{q} \in \mathbb{R}^{2}$, com $\mathbf{p}<\mathbf{q}$ (desigualdade em cada cordenada, $\mathbf{p} \neq \mathbf{q}$ ), seja $\Pi(\mathbf{p}, \mathbf{q})$ o conjunto de todos os caminhos crescentes em todas cordenadas, formados por pontos de $\mathbf{P}$, de $\mathbf{p}$ para $\mathbf{q}$, onde excluímos todos os pontos que possuem alguma cordenada em comum com p. Por caminho crescente de $\mathbf{P}$, entende-se um subconjunto ordenado $\left\{\left(a_{1}, b_{1}\right),\left(a_{2}, b_{2}\right), \ldots\left(a_{n}, b_{n}\right) \ldots\right\}$ de $\mathbf{P}$, em que $a_{1}>a_{2}>\ldots>a_{n} \ldots$ e $b_{1}>b_{2}>\ldots>b_{n} \ldots$ O tempo de última passagem entre $\mathbf{p} \leq \mathbf{q}$ é definido por

$$
T(\mathbf{p}, \mathbf{q}):=\max _{\varpi \in \Pi(\mathbf{p}, \mathbf{q})}\left\{\sum_{a \in \varpi} 1\right\}
$$

Esta variável mede o tamanho da maior sequência crescente entre $\mathbf{p}$ e $\mathbf{q}$, dentre as sequências crescentes formadas exclusivamente por pontos do processo $\mathbf{P}$.

Aldous e Diaconis [1] mostraram que o fluxo é equivalente à seguinte variável: Para cada $\nu \in \mathcal{X}, x \in \mathbb{R}$ e $t \geq 0$ seja

$$
L_{\nu}(x, t)=\sup _{z \leq x}\{\nu(z)+T((z, 0),(x, t))\}
$$

Assim, podemos ver o fluxo $L_{\nu}$ como sendo o tamanho da maior sequência crescente entre $\mathbf{p}$ e $\mathbf{q}$, dentre as possíveis sequências crescentes formadas por pontos do processo $\mathbf{P}$ e pontos da medida inicial $\nu$. A partir desta representação o processo de Hammersley $\left(M_{t}^{\nu}\right)$ pode ser redefinido por

$$
M_{t}^{\nu}((x, y]):=L_{\nu}(y, t)-L_{\nu}(x, t) \text { para } x<y
$$




\section{Processo de Hammersley em uma caixa finita}

Defina $\forall x \in \mathbb{R}$ e $t \geq 0$ :

$$
\begin{gathered}
N_{t}(x):=L_{\nu}((0, t),(x, t))=M_{t}^{\nu}(x) \\
S(x):=N_{0}(x)=L_{\nu}((0,0),(x, 0))=\nu(x), \\
E_{x}(t):=L_{\nu}((x, 0),(x, t)), \\
W(t):=E_{0}(t)=L_{\nu}((0,0),(0, t)),
\end{gathered}
$$

Da aditividade do fluxo, obtemos que $L_{\nu}(x, t)=S(x)+E_{x}(t)$. Isto nos induz a estudar o processo de Hammersley restrito à uma caixa finita cujos lados são os processos $N, S, E, W$ (ver figura 2.1) cujas letras fazem referência às cordenadas polares em inglês para $x>0$ (neste caso, $L_{\nu}(x, t)=S+E=W+N$ ). Nesse caso, observe que o fluxo é positivo, ie, se $x \geq 0$ então $L_{\nu}(x, t) \geq 0$.

Essa descrição também é conhecida como Processo de Hammersley com entradas e saídas ( ver Cator e Groeneboom [8] [9] para detalhes). Uma vantagem dela é explorar as simetrias do processo. Por exemplo, observe que se rotacionarmos o processo em $\theta=\frac{\pi}{2}$ radianos no sentido anti-horário obtemos um processo de Hammersley com as partículas saltando no sentido oposto, ie, da esquerda para a direita. Neste novo processo, tempo e espaço trocam de lugar. Se tomarmos $S$ como um processo de Poisson de taxa $\lambda$, então $E_{x}(t)$ tem lei Poisson de taxa $\frac{t}{\lambda}$ para todo $(x, t)[8]$.

Outra propriedade importante é que, se $\nu=\nu_{\lambda}$, para $x>0$ o processo $W(t)$ é independente de $S(x)$. Para $x<0, E_{x}(t)$ é independente de $S(x)$.

\section{Choque e resultados}

Nesta seção definimos o choque no processo de Hammersley e enunciamos os principais resultados da tese.

Considere dois processos de Hammersley em equilíbrio, um com medida inicial $\nu_{\lambda}$ e o outro com medida inicial $\nu_{\rho}$, onde $\lambda>\rho$. Estudaremos o processo no regime de choque, com medida inicial $\nu_{\rho, \lambda}$. Sua configuração à esquerda da origem coincide com $\nu_{\rho}$ e à direita coincide $\operatorname{com} \nu_{\lambda}$. Denotamos o processo de Hammersley com essa medida inicial por $\left(M_{t}^{\nu_{\rho, \lambda}}\right)_{t \geq 0}$. 


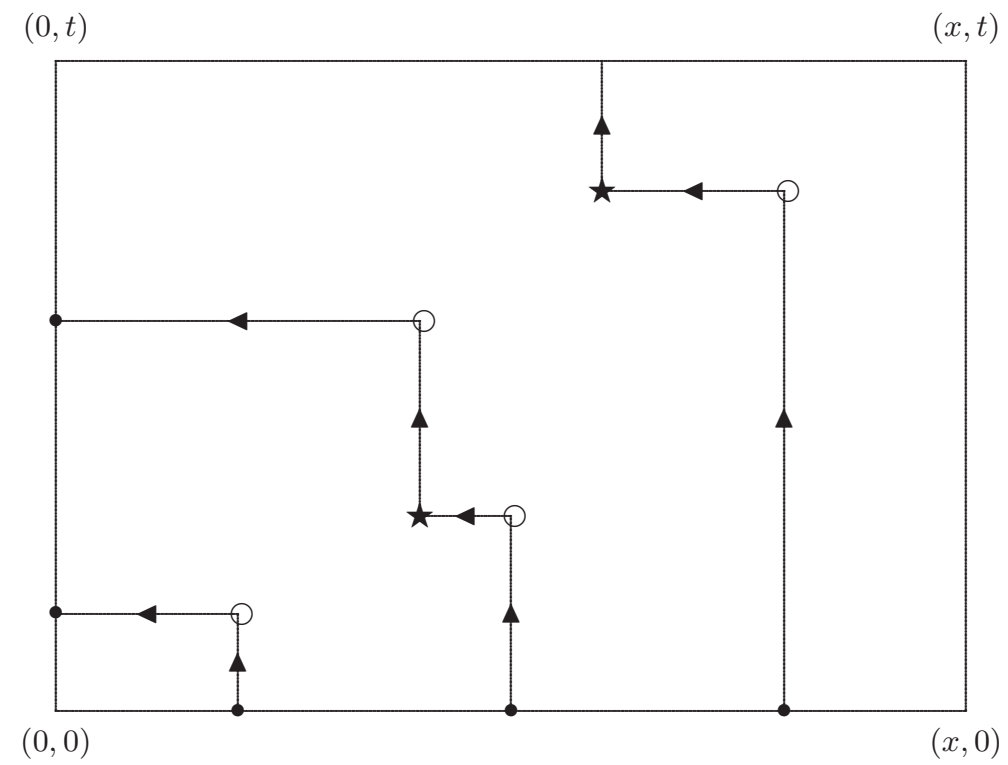

Figura 2.1: Trajetórias das partículas no processo de Hammersley restrito a caixa $[0, x] \times[0, t]$. Os pontos de $\mathbf{P}$ estão representados pelas estrelas e os pontos duais de $\mathbf{P}$ pelos círculos grandes.

Um choque microscópico relacionado ao processo $\left(M_{t}^{\nu}\right)_{t \geq 0}$ é uma posição (possivelmente) aleatória $Z_{t}^{\nu}$ tal que as densidades assintóticas à direita e à esquerda do processo $M_{t}^{\nu}$ translado por $Z_{t}^{\nu}$ são diferentes, uniformemente em t. Ferrari e Martin [16] deram uma construção elegante do choque baseada na versão multiclasse do processo de Hammersley para um número finito de classes de partículas. O processo de Hammersley multiclasse consiste numa convenção do acoplamento básico de dois ou mais processos de Hammersley com configurações iniciais ordenadas, ie, uma domina a outra. Aqui falaremos mais especificamente do caso onde temos duas classes de partículas. Para o caso em que há um número infinito de classes veja Cator e Pimentel [10]. Para realizar o acoplamento básico de dois ou mais processos de Hammersley utilizase o mesmo processo de Poisson bidimensional $\mathbf{P}$ para ambas configurações inicias. Em nosso caso, $\left(M_{t}^{\nu_{\lambda}}, M_{t}^{\nu_{\rho}}\right)_{t \geq 0}$ é o acoplamento de dois processos de Hammersley com medidas iniciais Poisson $\nu_{\lambda}$ e $\nu_{\rho}$ tais que para todo $y \in \mathbb{R}$ temos $\nu_{\lambda}(y) \geq \nu_{\rho}(y)$. O acoplamento básico é atrativo, o que significa que se uma das medidas iniciais domina a outra, então para todo tempo $t$ a dominação 
é preservada, isto é, para todo $t$ positivo e $y \in \mathbb{R}$ temos $M_{t}^{\nu_{\lambda}}(y) \geq M_{t}^{\nu_{\rho}}(y)$. Desta forma, as discrepâncias entre $M_{t}^{\nu_{\lambda}}$ e $M_{t}^{\nu_{\rho}}$ podem ser vistas como um processo conservativo de partículas que denotaremos por

$$
\left(\xi_{t}\right)_{t \geq 0}:=\left(M_{t}^{\nu_{\lambda}}-M_{t}^{\nu_{\rho}}\right)_{t \geq 0}
$$

Um ponto de $\xi_{t}$ é chamado de partícula de segunda classe que é uma discrepância marcada entre dois processos marginais do acoplamento básico. Elas se comportam como partículas (diferentes das partículas usuais) que saltam para a posição da partícula de primeira classe mais próxima a sua direita no momento em que esta salta para um ponto de $\mathbf{P}$ que está localizado à esquerda da posição da partícula de segunda classe. Denominamos o processo $\left(M_{t}^{\nu_{\rho}}, \xi_{t}\right)_{t \geq 0}$ por processo de duas classes.

Partículas de segunda classe são úteis para estudar o comportamento de perturbações microscópicas do sistema. Acoplando duas configurações iniciais que se diferenciam em apenas um ponto, a partícula de segunda classe, podemos ver como esta modificação afeta o sistema ao longo do tempo. Acontece que existe uma correspondência entre a posição de uma partícula de segunda classe colocada na origem de um sistema de choque e a posição de uma partícula de segunda classe colocada na origem de um processo de duas classes. Assim, para estudarmos o comportamento de uma perturbação num sistema de choque podemos estudar o comportamento de uma partícula de segunda classe no processo de duas classes correspondente. Para ver isso, observe que no processo de duas classes a trajetória de uma partícula de segunda classe não é afetada pelas partículas de segunda classe que estão atrás dela (a sua esquerda) e que a mesma trajetória não distingue as classes de partículas a sua direita. Isto pode ser formalizado acoplando-se as medidas iniciais do sistema de choque e do processos de duas classes, o que faremos no próximo capítulo, na proposição 3.1.

A partícula de segunda classe está relacionada com uma variável do modelo de percolação de última passagem, o ponto de saída. Definimos o ponto de saída $Y^{\nu}(x, t)$ para o maior caminho crescente da origem à $(x, t)$ como sendo $Y^{\nu}(x, t):=\sup \left\{z \leq x: \nu(z)+T((z, 0),(x, t))=L_{\nu}(x, t)\right\}$.

Considere a medida inicial de choque $\nu_{\rho, \lambda}$. Uma propriedade importante que 
o ponto de saída satisfaz é

$$
Y^{\nu_{\rho, \lambda}}(x, t) \stackrel{D}{=} x-Z_{t}^{\nu_{\rho, \lambda}}
$$

Uma prova desse fato pode ser obtida de modo análogo à prova de (5.6) contida no capítulo 5. Desse modo, obtemos resultados análogos aos do choque $Z_{t}^{\nu_{\rho, \lambda}}$ para o ponto de saída no modelo de percolação de última passagem com medida inicial $\nu_{\rho, \lambda}$.

Enunciaremos a diante os principais resultados da tese, que dizem respeito às flutuações dos fluxos de partículas de primeira e segunda classe e das partículas marcadas de primeira e segunda classe. Para estudar a posição das partículas marcadas utilizamos suas relações com os respectivos fluxos (veja Ferrari e Fontes [15] [14] para um método similar). Começamos no capítulo 4 com o seguinte resultado sobre o fluxo partículas de primeira classe no equilíbrio:

Teorema. 4.1 (Influência da condição inicial)

Seja $x \in \mathbb{R}$ e $t \geq 0$. Seja $L_{\lambda}(x, t)$ o fluxo da origem a $(x, t)$ em um processo de Hammersley com medida inicial $\nu_{\lambda}$ Poisson de taxa $\lambda$. Então

$$
\begin{aligned}
0 & <\liminf _{t \rightarrow \infty} \frac{\mathbb{E}\left(L_{\lambda}(x, t)-\nu_{\lambda}\left(x-\frac{t}{\lambda^{2}}\right)-\frac{2 t}{\lambda}\right)^{2}}{t^{\frac{2}{3}}} \\
& \leq \limsup _{t \rightarrow \infty} \frac{\mathbb{E}\left(L_{\lambda}(x, t)-\nu_{\lambda}\left(x-\frac{t}{\lambda^{2}}\right)-\frac{2 t}{\lambda}\right)^{2}}{t^{\frac{2}{3}}}<\infty
\end{aligned}
$$

A partir deste, obtemos como corolários teoremas centrais do limite para o fluxo $L_{\lambda}$ e para a partícula marcada de primeira classe $X_{t}$. Enunciamos o último a seguir:

Teorema. 4.2 Seja $X_{t}$ a posição no tempo $t$ de uma partícula marcada colocada na origem de um processo de Hammersley com medida inicial $\nu_{\lambda}$ Poisson de taxa $\lambda>0$. Temos:

$$
\lim _{t \rightarrow \infty} \mathbf{P}\left(X_{t} \leq-\frac{t}{\lambda^{2}}+\left(\frac{\sqrt{2 t}}{\sqrt{\lambda^{3}}}\right) u\right)=\mathbf{P}(N \leq u),
$$

onde $N$ é uma variável Normal padrão. 
Seja $L_{\xi}(x, t):=L_{\nu_{\lambda}}(x, t)-L_{\nu_{\rho}}(x, t)$ o fluxo de partículas de segunda classe da origem a $(x, t)$. Um dos principais ingredientes que utilizaremos nas provas dos teoremas a seguir é a seguinte relação

$$
L_{\xi}(x, t)= \begin{cases}-\xi_{t}\left(\left(x, Z_{0}(t)\right]\right) & \text { se } x<Z_{0}(t) \\ 0 & \text { se } x=Z_{0}(t) \\ \xi_{t}\left(\left(Z_{0}(t), x\right]\right) & \text { se } x>Z_{0}(t)\end{cases}
$$

Dela derivamos um teorema central do limite para o choque, provado no capítulo 4:

Teorema. 4.2 Seja $Z_{t}^{\nu_{\rho, \lambda}}$ a posição no tempo $t$ de uma partícula de segunda classe colocada na origem de um processo de Hammersley com medida inicial no regime de choque $\nu_{\rho, \lambda}$, Poisson de taxa $\rho$ à esquerda da origem e Poisson de taxa $\lambda$ à direita da origem, com $\rho<\lambda$. Então

$$
\lim _{t \rightarrow \infty} \mathbf{P}\left(Z_{t}^{\nu_{\rho, \lambda}} \leq \frac{t}{\lambda \rho}+(\eta \sqrt{t}) u\right)=\mathbf{P}(N \leq u)
$$

onde $N$ é uma variável Normal padrão e $\eta:=\frac{\sqrt{2}}{\sqrt{\lambda \rho} \sqrt{\lambda-\rho}}$.

No capítulo 5 utilizamos outra abordagem para explorar novamente a relação entre fluxo de partículas de segunda classe e a posição do choque para calcularmos o coeficiente de difusão do choque $D:=\lim _{t \rightarrow \infty} \frac{\operatorname{Var} Z_{t}^{\nu \rho, \lambda}}{t}$. Esta abordagem também é utilizada para a partícula de primeira classe, onde podemos obter os momentos para todo tempo $t$. Nos mostraremos que:

Teorema. 5.2 Seja $X_{t}$ a posição no tempo $t$ da partícula marcada colocada na origem no tempo 0 em um processo de Hammersley em equilíbrio com medida inicial $\nu$ Poisson de intensidade $\lambda$. Então para todo $t \geq 0$,

$$
\mathbb{E} X_{t}=-\frac{t}{\lambda^{2}}
$$

$e$

$$
\operatorname{Var} X_{t}=\frac{2 t}{\lambda^{3}}
$$


Teorema. 5.3 Seja $Z_{t}^{\nu_{\rho, \lambda}}$ a posição no tempo $t$ de uma partícula de segunda classe colocada na origem de um processo de Hammersley com medida inicial no regime de choque $\nu_{\rho, \lambda}$, Poisson de taxa $\rho$ à esquerda da origem e Poisson de taxa $\lambda$ à direita da origem, com $\rho<\lambda$. Para todo $t \geq 0$ e $\epsilon>0$,

$$
\operatorname{Var} Z_{t}^{\nu_{\rho, \lambda}}=\frac{2 t}{\lambda \rho(\lambda-\rho)}+o\left(t^{\epsilon+2 / 3}\right)
$$

Analogamente ao fluxo de partículas de primeira classe, podemos restringir o processo $\left(\xi_{t}\right)$ à uma caixa finita. Neste caso, para $x, t \geq 0$ temos

$$
L_{\xi}(x, t)=N_{\xi}+W_{\xi}=S_{\xi}+E_{\xi}
$$

onde $N_{\xi}:=N_{\lambda}(x)-N_{\rho}(x), S_{\xi}:=S_{\lambda}(x)-S_{\rho}(x), W_{\xi}:=W_{\lambda}(t)-W_{\rho}(t)$ e $E_{\xi}:=E_{\lambda}(t)-E_{\rho}(t)$.

Usaremos essa formulação do processo restrita à uma caixa finita para mostrar no capítulo 6 uma fórmula relacionando o fluxo de partículas de segunda classe no processo em equilíbrio multiclasse com a variância da partícula isolada de segunda classe no processo de Hammersley com uma classe em equilíbrio. A partir desta, obtemos o seguinte resultado:

Teorema. $6.2 \operatorname{Seja} L_{\xi}(x, t)$ o fluxo de partículas de segunda classe da origem a $(x, t)$ em um processo de duas classes em equilibrio. Seja $V>0$ uma constante e $x=x_{t}$ uma função de $t$. Se $\lim _{t \rightarrow+\infty} \frac{x_{t}}{t}=V$ entãa

$$
\operatorname{Var} L_{\xi}\left(x_{t}, t\right)=f(t)+g(t)+O(1)
$$


onde

$$
\begin{aligned}
f(t)= & f(V, t) \\
:= & (\lambda+\rho)\left(\frac{t}{\lambda \rho}-x_{t}\right)+ \\
& \quad+2\left(\lambda x_{t}-t / \lambda\right) \mathbb{P}\left(Z^{\nu_{1}}(t / \lambda) \leq \lambda x_{t}\right)+2\left(\rho x_{t}-t / \rho\right) \mathbb{P}\left(Z^{\nu_{1}}(t / \rho) \leq \rho x_{t}\right)
\end{aligned}
$$

é da ordem de $t$

$$
\begin{aligned}
& g(t)=g(V, t) \\
& :=2 \mathbb{E}\left(\left[t / \lambda-Z^{\nu_{1}}(t / \lambda)\right] \mathbf{1}_{\left[Z^{\left.\nu_{1}(t / \lambda) \leq \lambda x_{t}\right]}\right.}\right)+2 \mathbb{E}\left(\left[t / \rho-Z^{\nu_{1}}(t / \rho)\right] \mathbf{1}_{\left[Z^{\nu_{1}}(t / \rho) \leq \rho x_{t}\right]}\right)
\end{aligned}
$$

é da ordem de $t^{2 / 3}$.

Em particular,

$$
\lim _{t \rightarrow \infty} \frac{\operatorname{Var} L_{\xi}(V t, t)}{t}=\lim _{t \rightarrow \infty} \frac{f(t)}{t}= \begin{cases}(\lambda+\rho)\left(\frac{1}{\lambda \rho}-V\right) & \text { se } V \leq \frac{1}{\lambda^{2}} \\ (\lambda-\rho)\left(\frac{1}{\lambda \rho}+V\right) & \text { se } V \in\left(\frac{1}{\lambda^{2}}, \frac{1}{\rho^{2}}\right) \\ (\lambda+\rho)\left(V-\frac{1}{\lambda \rho}\right) & \text { se } V \geq \frac{1}{\rho^{2}}\end{cases}
$$

\section{Processo multiclasse}

Extenderemos agora a definição do processo de Hammersley para um número de classes igual a $n \geq 2$. Como no processo de duas classes, utilizamos o acoplamento básico para realizar diferentes configurações iniciais do processo no mesmo espaço de probabilidade induzido pelo processo de Poisson bidimensional $P$. Agora, acoplamos $n$ medidas iniciais $\nu_{1}, \ldots, \nu_{n}$, com $n \geq 2$, tais que para todo $x \in \mathbb{R}, \nu_{1}(\{x\}) \leq \ldots \leq \nu_{n}(\{x\})$. Se $\nu_{n}(\{x\})=1$, dizemos que $x$ possui uma partícula de classe $k \in\{1, \ldots n\} \operatorname{se}_{i \in\{1, \ldots, n\}}\left\{i: \nu_{i}(\{x\})=1\right\}=k$. Com esta definição, vemos que as partículas de classe $k$ podem ser ultrapassadas pelas partículas de menor classe e podem ultrapassar partículas de maior classe.

Sejam

$$
\mathcal{X}_{n \uparrow}=\left\{\left(\nu_{1}, \ldots, \nu_{n}\right) \in \mathcal{X}_{n}: \nu_{1}(\{x\}) \leq \ldots \leq \nu_{n}(\{x\}) \text { para todo } x \in \mathbb{R}\right\}
$$


o espaço das configurações ordenadas do acoplamento básico de processos HAD em $\mathbb{R}$ e $\mathcal{X}_{n}$ o espaço das configurações bem definidas do acoplamento de $\mathrm{n}$ processos de Hammersley na reta. Definimos o processo Hammersley multiclasse de forma precisa como

$$
\psi_{t}=R \nu_{t}
$$

em que a bijeção $R: \mathcal{X}_{n \uparrow} \rightarrow \mathcal{X}_{n}$ é definada por

$$
(R \nu)_{k}=\nu_{k} \backslash \nu_{k-1}
$$

para todo $k \in\{1, \ldots, n\}$. Assim, $\psi_{t}=\left(M_{t}^{\psi^{1}}, \ldots, M_{t}^{\psi^{n}}\right)$, onde $M_{t}^{\psi^{i}}$ é a configuração das partículas de i-ésima classe no tempo $t$.

Partículas marcadas de classes maiores do que 2 podem ser vistas como partículas de segunda classe no choque quando olhamos para o processo $\left(M_{t}^{\psi^{i}-1}, M_{t}^{\psi^{i}}\right)_{t \geq 0}$ formado pelas marginais $i-1$ e $i$ do processo multiclasse $M_{t}^{\psi}$. Assim, todos os resultados a respeito de partículas de segunda classe no processo de duas classe que descrevemos na seção anterior se extendem para partículas de classes maiores do que 2 . 


\section{Capítulo 3}

\section{Preliminares}

Neste capítulo mostraremos algumas propriedades que serão usadas nas demonstrações dos teoremas principais.

\section{Choque e processo de duas classes no equilíbrio}

Começaremos descrevendo o processo de Hammersley de duas classes $\left(M_{t}^{\nu_{\rho}}, \xi_{t}\right)_{t \geq 0}$ no regime de equilíbrio. Indexamos as partículas de segunda classe no tempo zero começando com a primeira partícula à esquerda da origem que recebe o índice zero. As partículas à direita são numeradas com os inteiros positivos de 1 em diante e, da mesma forma, as partículas à esquerda são numeradas com os inteiros negativos. Denotamos por $Z_{i}(t)$ a posição no tempo $t$ da iésima partícula de segunda classe marcada, para $t \geq 0$.

A medida invariante para o processo de duas classes pode ser vista como a saída de uma fila $M / M / 1$ em equilíbrio. A fila $M / M / 1$ é um processo de Markov bem conhecido que modela uma fila com um único servidor, onde clientes chegam um por um de acordo com um processo de Poisson de taxa $\mu_{1}$ e os tempos entre os serviços são independentes e identicamente distribuídos com lei exponencial de taxa $\mu_{2}$. Denotamos por $Q(s)$ o número de clientes na fila no tempo $s \in \mathbb{R}$. Se $\mu_{1}<\mu_{2}$, então existe uma única medida invariante para o processo $(Q(s))_{s \in \mathbb{R}}$, que é finita com probabilidade 1 e reversível. Este processo pode ser construído como função de dois processos de Poisson independentes, o processo de chegadas $(A(s))_{s \in \mathbb{R}}$ de taxa $\mu_{1}$ e o processo de saídas $(D(s))_{s \in \mathbb{R}}$ de taxa $\mu_{2}$. Suponha que no tempo $r$ haja $n$ clientes na fila, ie, $Q(r)=n$. Quando o primeiro ponto de $A(r-s)$ é alcançado para um $s$ positivo, um cliente chega a fila e $Q(r-s)=Q(r)+1$. Observamos que nesse caso, o tempo $s$ da fila está decrescendo (podemos fazer isso, pois a fila é um processo reversível). Isto será conveniente mais adiante. Quando um ponto de $(D()$.$) é alcançado, então ou$ 
um cliente da fila é servido e deixa a fila ou se a fila estiver vazia um serviço possível não será utilizado.

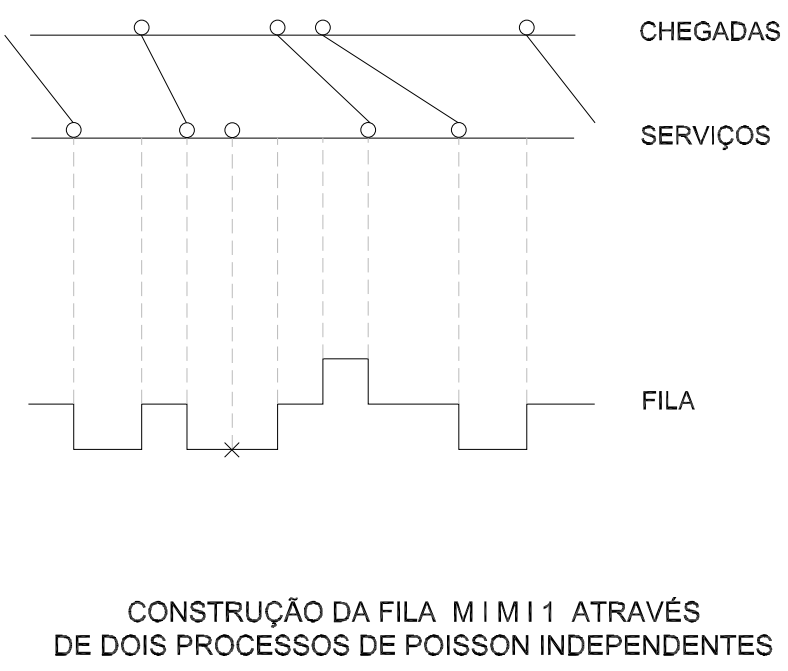

O processo de saídas $(D()$.$) é a medida invariante para o processo de Ham-$ mersley com duas classes de partículas, onde os serviços não utilizados são as partículas de segunda classe e os serviços efetivamente utilizados são as partículas de primeira classe. Além disso, é possível construir toda uma realização do processo de duas classes em equilíbrio usando a mesma função acima no acoplamento chamado de processo multilinha.

O processo multilinha é um acoplamento $\left(M_{t}^{\alpha_{1}}, M_{t}^{\alpha_{2}}, \ldots, M_{t}^{\alpha_{n}}, \mathbf{P}\right)$ (diferente do acoplamento básico) de $n$ processos de Hammersley. Aqui, nos deteremos ao caso $n=2$. Acoplamos dois processos de Hammersley $\left(M_{t}^{\alpha_{1}}\right)_{t \geq 0}$ e $\left(M_{t}^{\alpha_{2}}\right)_{t \geq 0}$ com medidas inicias $\alpha_{1}$ e $\alpha_{2}$, do seguinte modo: a segunda marginal tem sua dinâmica de saltos determinada pelo processo de Poisson bidimensional $\mathbf{P}$, enquanto a primeira marginal tem sua dinâmica de saltos determinada pelo processo $d(\mathbf{P})$ dos pontos duais de $\mathbf{P}$. Os pontos duais $d(\mathbf{P})$ são as posições onde haviam uma partícula do processo $\left(M_{t}^{\alpha_{2}}\right)_{t \geq 0}$ imediatamente antes desta mesma partícula saltar sobre um ponto de $\mathbf{P}$ (veja a figura 2.1). Cator e Groeneboom [8] mostraram um teorema de Burke para $\mathbf{P}$ provando que $d(\mathbf{P})$ também é um processo de Poisson bidimensional de taxa 1 , o que mostra que $\left(M_{t}^{\alpha_{1}}\right)_{t \geq 0}$ é de fato um processo de Hammersley. O produto de processos de Poissons é a única medida ergódica invariante para o processo multilinha.

Existe uma correspondência biunívoca entre um processo multiclasse e um processo multilinha. Considere um processo multilinha com medida inicial pro- 
duto de Poissons independentes. Como o produto de Poissons independentes é medida invariante para o processo multilinha, então se aplicarmos a função que leva o processo multilinha em equilíbrio no processo multiclasse correpondente, obteremos a medida invariante para o processo multiclasse. Tal medida invariante pode ser interpretada como a sáida de uma fila.

Novamente, fixemos o caso com duas classes de partículas. Sejam $\alpha_{1}$ e $\alpha_{2}$ dois processos de Poisson em $\mathbb{R}$ independentes com taxas $\lambda$ e $\rho$ respectivamente, onde $\lambda>\rho$. Considere o processo multilinha $\left(M_{t}^{\alpha_{1}}, M_{t}^{\alpha_{2}}, \mathbf{P}\right)$ com medidas iniciais $\alpha_{1}$ e $\alpha_{2}$. Como visto no começo da seção, podemos construir uma fila $M / M / 1$ estacionária a partir de $\alpha_{1}$ e $\alpha_{2}$, onde colocamos $\alpha_{1}$ como o processo de chegadas de clientes da fila e $\alpha_{2}$ como o processo de serviços da fila. Agora, como esta medida é invariante para o processo multilinha, então, para todo tempo $t$, podemos construir uma fila $\left(Q_{t}(x)\right)_{x \in \mathbb{R}}$ a partir dos processos $M_{t}^{\alpha_{1}} \mathrm{e}$ $M_{t}^{\alpha_{2}}$ se colocarmos $\left(M_{t}^{\alpha_{1}}(x)\right)_{x \in \mathbb{R}}$ como o processo de chegadas e $\left(M_{t}^{\alpha_{2}}(x)\right)_{x \in \mathbb{R}}$ como o processo de saídas (observe que o tempo da fila é o espaço do processo de Hammersley). O teorema de Burke para filas $M / M / 1$ nos diz que os serviços efetivamente utilizados na fila $\left(Q_{t}(x)\right)_{x \in \mathbb{R}}$ formam um processo de Poisson em $\mathbb{R}$ de taxa $\rho$, que denotaremos por $M_{t}^{\nu_{\rho}}$. Denotaremos os serviços não utilizados da fila $\left(Q_{t}(x)\right)_{x \in \mathbb{R}}$ por $\xi_{t}$.

Ferrari e Martin [16] provaram que usando a fila $\left(Q_{t}(x)\right)_{x \in \mathbb{R}}$ construída a partir de $\left(M_{t}^{\alpha_{1}}(x)\right)_{x \in \mathbb{R}}$ e $\left(M_{t}^{\alpha_{2}}(x)\right)_{x \in \mathbb{R}}$ teremos um processo de duas classes em equilíbrio $\left(M_{t}^{\nu_{\rho}}, \xi_{t}\right)$. Isto é, seja $\Phi$ a função que indexa as partículas de $M_{t}^{\alpha_{2}}$ como partículas de primeira ou segunda classe de acordo com a fila $Q_{t}$ construída como uma função $\Psi$ do processo multilinha $\left(M_{t}^{\alpha_{1}}, M_{t}^{\alpha_{2}}, \mathbf{P}\right)$, temos que

$$
\left(M_{t}^{\nu_{\rho}}, \xi_{t}\right)=\Phi\left(Q_{t}\right)=\Phi\left(\Psi\left(\left(M_{t}^{\alpha_{1}}, M_{t}^{\alpha_{2}}, \mathbf{P}\right)\right)\right)
$$

é de fato um processo de Hammersley de duas classes e, neste caso, sabemos que sua medida inicial é invariante (veja [16] para mais detalhes).

Agora vamos mostrar que a descrição acima da medida invariante do processo de duas classes implica que tanto a partícula marcada de segunda classe $Z_{0}(t)$ no processo de duas classes no equilíbrio como a partícula marcada de segunda classe $Z_{t}^{\nu_{\rho, \lambda}}$ no processo de Hammersley clássico com medida inicial $\nu_{\rho, \lambda}$ são choques microscópicos. 
Proposição 3.1. Considere um processo de duas classes no regime de equilíbrio com densidades $\rho$ e $\lambda-\rho$ de partículas de primeira e segunda classe respectivamente, onde $\lambda>\rho>0$. Seja $\left(M_{t}^{\nu_{\rho}}, \xi_{t}^{\prime}\right)_{t \geq 0}$ este processo condicionado em haver uma partícula de segunda classe na origem. Seja $Z_{0}(t)$ a posição no tempo $t$

da partícula de $\xi_{0}^{\prime}$ que se encontra na origem no tempo 0. Agora, seja $Z_{t}^{\nu_{\rho, \lambda}}$ a posição no tempo t de uma partícula de segunda classe colocada na origem de um processo de Hammersley clássico com medida inicial $\nu_{\rho, \lambda}$. Temos que ambas, $Z_{0}(t)$ e $Z_{t}^{\nu_{\rho, \lambda}}$, são choques microscópicos. Além disso, é possível acoplar os processos de tal modo que, para todo $t$

$$
\left|Z_{t}^{\nu_{\rho, \lambda}}-Z_{0}(t)\right| \leq J_{t}
$$

onde $\left(J_{t}\right)_{t \leq 0}$ é uma familía de variáveis aleatórias identicamente distribuídas, cuja lei tem todos os momentos finitos.

\section{Prova da Proposição 3.1}

Considere o processo de duas classes $\left(M_{t}^{\nu_{\rho}}, \xi_{t}^{\prime}\right)_{t \geq 0}$ com medida inicial invariante condicionada em haver uma partícula de segunda classe $Z_{0}$ na origem. Considere que o mesmo processo foi construído como uma função de um processo multilinha $\left(M_{t}^{\alpha_{1}}, M_{t}^{\alpha_{2}}, \mathbf{P}\right)$. Seja $\left(Q_{0}(x)\right)_{x \in \mathbb{R}}$ a fila $M / M / 1$ gerada por $\alpha_{1}$ e $\alpha_{2}$ e que da origem à medida inicial $\left(M_{0}^{\nu_{\rho}}, \xi_{0}^{\prime}\right)$. Como condicionamos em haver uma partícula de segunda classe na origem, isto implica que $Q_{0}(0)=0$.

Agora vamos verificar como é o processo visto pela partícula de segunda classe $Z_{0}(t)$. A partícula de segunda classe tem sua dinâmica afetada do mesmo modo por partículas que estão à sua direita, independente de serem partículas de primeira classe ou de segunda classe. Isto é, se alteramos a classe de uma partícula que se encontra à direita de $Z_{0}(t)$, então sua trajetória não é afetada. Deste modo, do ponto de vista de $Z_{0}(t)$, podemos ver todas as partículas à sua direita como partículas de primeira classe. Pela construção da fila, obtemos que $Z_{0}$ enxerga um processo de Poisson de taxa $\lambda$ à sua direita (o processo $\alpha_{2}$ ). À esquerda de $Z_{0}$, apenas as partículas de primeira classe afetam sua trajetória, ie, acrescentando ou retirando partículas de segunda classe que estão à esquerda de $Z_{0}(0)$ a trajetória de $Z_{0}(t)$ não é afetada. Pela construção da fila, obtemos que $Z_{0}$ enxerga à sua esquerda o processo das saídas dos clientes de uma fila $M / M / 1$ que no tempo zero está vazia. Este processo não é Poisson, mas vamos mostrar que ele se assemelha muito a um processo de Poisson de taxa $\rho$.

Para ver isso, vamos construir uma nova fila $\left(G_{0}(x)\right)_{x \in \mathbb{R}}$ cujo processo de 
chegadas também é $\alpha_{1}$ e o processo de saídas também é $\alpha_{2}$, mas $G_{0}(0)$ é uma variável aleatória com distribuição geométrica de taxa $\frac{\rho}{\lambda}$ independente dos processos $\alpha_{1}$ e $\alpha_{2}$. Essa nova fila é estacionária, e portanto satisfaz o teorema de Burke para filas $M / M / 1$. Isto quer dizer que o processo de saídas dos clientes da fila $G_{0}$ é um processo de Poisson de taxa $\rho$. Assim, fixe uma realização de $G_{0}(0)=k, \alpha_{1}$ e $\alpha_{2}$. Com probabilidade $1, k<\infty$ e o processo de saídas de clientes da fila $\left(G_{0}(x)\right)_{x \leq 0}$, com o tempo decrescendo, coincide com $M_{0}^{\nu_{\rho}} \cup \xi_{0}^{\prime}$ até o k-ésimo ponto de $\xi_{0}^{\prime}$, ie, até que apareça a k-ésima partícula de segunda classe na medida inicial do processo multiclasse (na direção de 0 a $-\infty$ ). A partir deste momento, ambas as filas $G_{0}$ e $Q_{0}$ terão sempre os mesmos valores, ie, para todo $x \leq Z_{-k}$ temos $G_{0}(x)=Q_{0}(x)$ e portanto, $M_{0}^{\nu_{\rho}}\left(-\infty, Z_{-k}(0)\right)$ é igual ao processo de Poisson de taxa $\rho$ gerado a partir da dos serviços utilizados de $\left(G_{0}(x)\right)_{x \leq 0}$. Por fim, observamos que $\left|Z_{-k}(0)\right|$ é finito com probabilidade 1.

Agora, as partículas de segunda classe são pontos de renovação para o processo multiclasse invariante. Isto implica que o processo como visto pela partícula de segunda classe gerado apartir de uma medida invariante multiclasse se encontra em equilíbrio (ver [16]). Portanto, para todo tempo $t$, podemos construir o processo visto por $Z_{0}(t)$ a partir da fila $\left(Q_{t}(x)\right)_{x \in \mathbb{R}}$ gerada por $M_{t}^{\alpha_{1}}$ e $M_{t}^{\alpha_{2}}$ e da mesma forma acoplarmos com a saída de uma fila $M / M / 1$ estacionária $\left(G_{t}(x)\right)_{x \in \mathbb{R}}$ e obtermos o mesmo resultado. Como $Z_{0}(t)-Z_{-k}(t)$ tem a mesma lei de $\left|Z_{-k}(0)\right|$, temos que

$$
\begin{aligned}
\frac{\mathbb{E}\left(Z_{0}(t)-Z_{-k}(t)\right)}{x} & =\frac{\left|\mathbb{E} Z_{-k}(0)\right|}{x} \\
& =\frac{c}{x}
\end{aligned}
$$

Assim, fazendo $x$ tender a menos infinito, obtemos que a densidade à esquerda de $Z_{0}(t)$ tende a mesma que à esquerda de $Z_{-k}(0)$, isto é $\rho$, independente de $t$. Logo $Z_{0}(t)$ é um choque microscópico associado ao processo $\left(M_{t}^{\nu_{\rho}}, \xi_{t}^{\prime}\right)_{t \geq 0}$.

Agora, vamos construir a medida $\nu_{\rho, \lambda}$ da seguinte forma: à esquerda da origem, ela coincide com os tempos de serviços utilizados de fila $\left(G_{0}(x)\right)_{x<0} \mathrm{e}$ à direita da origem ela coincide com a saída da fila $\left(G_{0}(x)\right)_{x>0}$, ie, coincide com $\alpha_{2}$. Na origem, colocamos uma partícula de segunda classe, cuja posição no tempo t é denotada por $Z_{t}^{\nu_{\rho, \lambda}}$. Realizamos o processo de Hammersley clássico $\left(M_{t}^{\nu_{\rho, \lambda}}\right)_{t \geq 0}$ utilizando o mesmo processo de saltos $\mathbf{P}$ utilizado no processo multilinha $\left(M_{t}^{\alpha_{1}}, M_{t}^{\alpha_{2}}, \mathbf{P}\right)$. Deste acoplamento, obtemos que: 
Para $x>0$

$$
\left|\nu_{\rho, \lambda}(-x)\right| \geq\left|M_{0}^{\nu_{\rho}}(-x)\right|
$$

e

$$
\left|\nu_{\rho, \lambda}(x)\right|=\alpha_{2}(x)
$$

o que implica que para todo $t$

$$
Z_{t}^{\nu_{\rho, \lambda}} \leq Z_{0}(t)
$$

Dado $G_{0}(0)=k<\infty$, acrescentamos uma partícula de segunda classe a $\nu_{\rho, \lambda}$ em $Z_{-k-1}(0)$. Observe que isto não afeta $Z_{t}^{\nu_{\rho, \lambda}}$ e, denotando por $Z_{G}(t)$ a posição no tempo $t$ desta partícula de segunda classe, temos que $Z_{G}(t)=Z_{-k-1}(t)$ para todo $t$. Como $Z_{t}^{\nu_{\rho, \lambda}} \geq Z_{G}(t)$, segue que

$$
Z_{-k-1}(t) \leq Z_{t}^{\nu_{\rho, \lambda}} \leq Z_{0}(t)
$$

Da onde concluimos que $Z_{t}^{\nu_{\rho, \lambda}}$ é um choque microscópico associado ao processo $\left(M_{t}^{\nu_{\rho, \lambda}}\right)_{t \geq 0}$.

Por fim, para todo $t \geq 0$, seja $J_{t}=Z_{0}(t)-Z_{-k-1}(t)$. Da equação (3.3) obtemos que $\left|Z_{t}^{\nu_{\rho, \lambda}}-Z_{0}(t)\right| \leq J_{t}$. Como o processo multiclasse se encontra em equilíbrio, segue que a familía de variáveis $\left(J_{t}\right)_{t \geq 0}$ é identicamente distribuída com a mesma lei de $\left|Z_{-k-1}(0)\right|$, que é finita com probabilidade 1 e possui todos os momentos finitos.

\section{Fluxo de partículas de segunda classe no equilíbrio}

Uma propriedade dos processos $E_{\xi}$ e $W_{\xi}$ é conservar a monotonicidade da medida inicial. Vale que

Proposição 3.2. Seja $\lambda>\rho>0$. Se $S_{\xi}([z, x]) \geq 0$ para todo $z \leq x$, então $E_{\xi}([r, t]) \leq 0$ para todo $r \leq t$. Em particular, $W_{\xi}([r, t]) \leq 0$ para todo $r \leq t$.

\section{Prova da Proposição 3.2}

Considere o acoplamento básico $\left(M_{t}^{\mu_{\lambda}}, M_{t}^{\mu_{\rho}}\right)_{t \geq 0}$ de dois processos de Hammersley definidos em $\mathbb{R}$ com densidades $\lambda$ e $\rho$, onde $\mu_{\lambda} \geq \mu_{\rho}$, ie, para todo $y \leq x$ 
$S_{\lambda}([y, x]) \geq S_{\rho}([y, x])$.

Seja $\bar{t}=\inf \left\{t \geq 0: E_{\lambda}([0, t])>0\right\}$. É suficiente mostrarmos que $E_{\rho}([0, \bar{t}])>$ 0 . Seja $y<0$ a posição da partícula imediatamente à esquerda da origem no tempo $\bar{t}$ no processo $\left(M_{t}^{\mu_{\lambda}}\right)_{t \geq 0}$. Como $M_{\bar{t}_{-}}^{\mu_{\lambda}} \geq M_{\bar{t}_{-}}^{\mu_{\rho}}$, temos que $M_{\bar{t}_{-}}^{\mu_{\rho}}(y)=0$. Assim, a primeira partícula à direita da origem no processo $\left(M_{t}^{\mu_{\rho}}\right)_{t \geq 0}$ é que salta para o ponto $y$ no tempo $\bar{t}$. Logo, $E_{\rho}([0, \bar{t}]) \geq 1$ e segue o resultado.

Um importante corolário da proposição acima é que as medidas invariantes dos fluxos verticais $E_{\xi}$ e $W_{\xi}$ também podem ser construídas como saídas de filas.

Corolário 3.1. Seja $\left.\left(N_{\phi}^{t}\right)\right)_{t \geq 0}$ um processo de Hammersley multiclasse clássico com 2 classes de partículas com medida invariante de taxas positivas $\left(\lambda_{1}, \lambda_{2}\right)$ para partículas de primeira e segunda classe respectivamente. $E_{\phi}^{x}(t)$ o fluxo multiclasse pelo ponto $x \in \mathbb{R}$ no tempo $t>0$ para este processo. Temos que $E_{\phi}^{x}(t)$ pode ser representado como a saída de uma fila $M / M / 1$ da mesma forma que $N_{\phi}^{t}$ mas com taxas $\left(\frac{1}{\lambda_{1}}-\frac{1}{\lambda_{1}+\lambda_{2}}, \frac{1}{\lambda_{1}+\lambda_{2}}\right)$.

\section{Prova do Corolário 3.1.}

Pela definição de processo multiclasse, sabemos que existe um processo acoplado $N_{\psi}^{t}=\left(N_{1}^{t}, N_{2}^{t}\right)$ que gera univocamente o processo multiclasse, cujas marginais têm densidades $\left(\lambda_{1}, \lambda_{1}+\lambda_{2}\right)$ e satisfaz $N_{1}^{t} \leq N_{2}^{t}$. Como o processo multiclasse $\left.\left(N_{\phi}^{t}\right)\right)_{t \geq 0}$ é invariante, então a lei de cada marginal do processo $N_{\psi}^{t}=$ $\left(N_{1}^{t}, N_{2}^{t}\right)$ é Poisson. Logo as marginais do fluxo por x, $E_{\phi}^{x}(t)=\left(E_{1}^{x}(t), E_{2}^{x}(t)\right)$, também são Poisson com taxas $\left(\frac{1}{\lambda_{1}+\lambda_{2}}, \frac{1}{\lambda_{1}}\right)$ (ver [9]). Como $N_{1}^{t} \leq N_{2}^{t}$, segue da proposição 3.2 que $E_{2}^{x}(t) \leq E_{1}^{x}(t)$. Desta forma, o processo $\left(E_{2}^{x}(t), E_{1}^{x}(t)\right)_{x \in \mathbb{R}}$ é um processo acoplado em equilíbrio que gera univocamente um processo multiclasse de taxas $\left(\frac{1}{\lambda_{1}}-\frac{1}{\lambda_{1}+\lambda_{2}}, \frac{1}{\lambda_{1}+\lambda_{2}}\right)$.

\section{Medida invariante multiclasse}

Nesta seção daremos uma descrição das medida invariantes do processo multiclasse com $n$ classes de partículas.

As únicas medidas invariantes extremas são obtidas como a saída de um sis- 
tema de $n-1$ filas em série com $n$ classes de clientes. A descrição do sistema é a seguinte: clientes de primeira classe chegam à primeira fila como um processo de Poisson de taxa $\rho_{1}$. Para cada fila $k$ com $k$ de 1 a $n-1$, existe uma quantidade infinita de clientes de classe $k+1$ esperando por atendimento. Quando um cliente de classe $i$ é atendido na fila $k$, ele passa para a próxima fila ou sai do sistema caso $k=n-1$. O atendimento segue a regra FIFO (First in first out) para clientes de mesma classe, ou seja, o primeiro a chegar é o primeiro a sair. Clientes de classe $i$ têm prioridade no atendimento com relação a clientes de classes maiores. Assim, por exemplo, quando um cliente de segunda classe chega na segunda fila, ele é atendido antes dos clientes de terceira classe que lá estavam. Por fim, as taxas de serviço do servidor da fila $k$ são exponenciais independentes de parâmetros $\rho_{k}$. Como todas as filas sempre possuem clientes esperando por atendimento, temos que os processos de saídas são processos de Poisson com taxas $\rho_{2}, \ldots \rho_{n}$. De imediato, aplicando o teorema de Burke sucessivas vezes, obtemos que a distribuição marginal dos clientes de primeira classe é um processo de Poisson de densidade $\rho_{1}$. O mesmo não ocorre para os clientes das outras classes, já que as chegadas deles no sistema não é um processo de Poisson homogêneo. Podemos ver que os clientes de classe $k$ (de 2 a $n$ ) chegam na fila $k+1$ nos momentos dos serviços não utilizados pelos clientes de classe menor que $k$. Podemos observar que nesta medida, clientes de classe maior que 1 não estão distribuídos uniformemente pelo espaço, o que ocorre com os clientes de primeira classe. Eles possuem uma certa tendência de se agrupar, formando pequenos clusters cujos tamanhos têm distribuição geométrica.
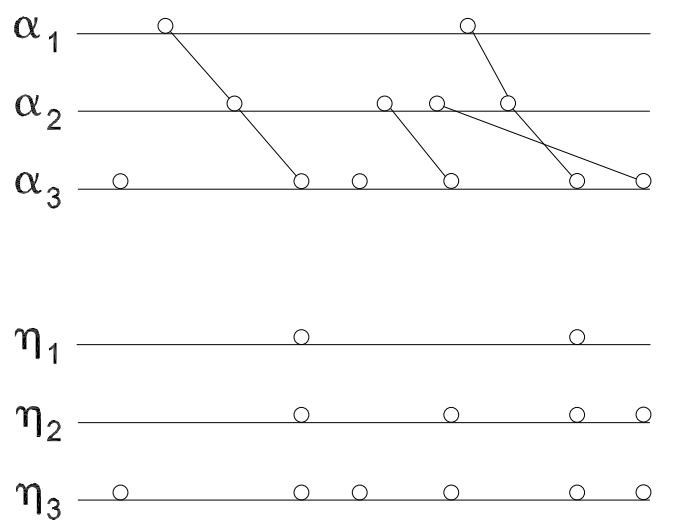
Para provar que de fato a medida acima é invariante para o processo $\psi_{t}$, Ferrari e Martin [16] utilizaram o Processo Multilinha. Seja $\alpha_{t}=\left(M_{t}^{\alpha_{1}}, \ldots, M_{t}^{\alpha_{n}}\right)$, onde as margimais são processos de Hammersley acoplados da seguinte maneira: $M_{t}^{\alpha_{n}}$ é governado pelo processo de Poisson bidimensional $P$ de taxa 1 , que é independente da configuração inicial $\alpha=\alpha_{0}$. Agora, denotando por $D\left(\mathbf{P}_{i}\right)$ os pontos duais do processo $M_{t}^{\alpha_{i}}$, temos que para todo $i \in\{1, \ldots, n-1\}, M_{t}^{\alpha_{i}}$ é governado pelos pontos $D\left(\mathbf{P}_{i+1}\right)$, definidos sucessivamente para i de $\mathrm{n}$ até 2 . O teorema de Burke garante que os processos $D\left(\mathbf{P}_{i+1}\right)$ são Poisson bidimensionais de taxa $1 \mathrm{e}$, portanto, as marginais $M_{t}^{\alpha_{1}}, \ldots, M_{n}^{\alpha_{1}}$ são de fato processos de Hammersley sempre que a configuração inicial de $\alpha$ for um produto de processos de Poisson homogêneos. 


\section{Capítulo 4}

\section{Teorema Central do Limite}

O principal resultado deste capítulo é um teorema central do limite para o choque microscópico $Z_{t}^{\nu_{\rho, \lambda}}$. A prova se baseia no fato que as flutuações de ordem 2 do fluxo de partículas $L_{\lambda}$ dependem apenas da condição inicial na escala difusiva $\sqrt{t}$. Este fato segue como corolário de um importante resultado de Cator e Groeneboom [9] e da proposição à seguir. Uma versão para o modelo de percolação de última passagem pode ser vista em [11].

Proposição 4.1. Seja $\left(M_{t}^{\nu}\right)_{t \geq 0}$ um processo de Hammersley com medida inicial $\nu$ e cuja dinâmica é determinada pelo processo de Poisson bidimensional $\mathbf{P}$ de taxa 1. Suponha $\nu$ invariante por translação e que $\nu$ e $\mathbf{P}$ são independentes. Então, para toda velocidade $V \in \mathbb{R}$ temos

$$
L_{\nu}(x, t)-\nu(x-V t) \stackrel{D}{=} L_{\nu}(V t, t)
$$

em lei.

Prova :

Segue da definição que o fluxo é aditivo. Assim, como $\nu$ também é aditiva, temos

$$
\begin{aligned}
L_{\nu}(x, t) & =\nu(x)+L_{\nu}((x, 0),(x, t)) \\
& =\nu(x-V t)+\nu((x-V t, x])+L_{\nu}((x, 0),(x, t))
\end{aligned}
$$

da onde

$$
L_{\nu}(x, t)-\nu(x-V t)=\nu((x-V t, x])+L_{\nu}((x, 0),(x, t))
$$


Pela invariância por translações de $\nu$ e de $\mathbf{P}$ e pela independência destes, segue que

$$
\begin{aligned}
L_{\nu}(x, t)-\nu(x-V t) & =\nu((x-V t, x])+L_{\nu}((x, 0),(x, t)) \\
& \stackrel{D}{=} \nu(V t)+L_{\nu}((V t, 0),(V t, t)) \\
& =L_{\nu}(V t, t)
\end{aligned}
$$

onde a igualdade do meio é em distribuição.

Cator e Groeneboom [9] provaram que a ordem correta da variância do fluxo ao longo da direção característica é $t^{2 / 3}$, mais precisamente vale que

$$
0<\liminf _{t \rightarrow \infty} \frac{\operatorname{Var} L_{\lambda}\left(\frac{t}{\lambda^{2}}, t\right)}{t^{\frac{2}{3}}} \leq \limsup _{t \rightarrow \infty} \frac{\operatorname{Var} L_{\lambda}\left(\frac{t}{\lambda^{2}}, t\right)}{t^{\frac{2}{3}}}<\infty
$$

Assim, obtemos como corolários da proposição (4.1) os seguintes resultados:

Teorema 4.1. (Influência da condição inicial)

Seja $x \in \mathbb{R}$ e $t \geq 0$. Seja $L_{\lambda}(x, t)$ o fluxo da origem a $(x, t)$ em um processo de Hammersley com medida inicial $\nu_{\lambda}$ Poisson de taxa $\lambda$. Então

$$
\begin{aligned}
0 & <\liminf _{t \rightarrow \infty} \frac{\mathbb{E}\left(L_{\lambda}(x, t)-\nu_{\lambda}\left(x-\frac{t}{\lambda^{2}}\right)-\frac{2 t}{\lambda}\right)^{2}}{t^{\frac{2}{3}}} \\
& \leq \limsup _{t \rightarrow \infty} \frac{\mathbb{E}\left(L_{\lambda}(x, t)-\nu_{\lambda}\left(x-\frac{t}{\lambda^{2}}\right)-\frac{2 t}{\lambda}\right)^{2}}{t^{\frac{2}{3}}}<\infty
\end{aligned}
$$

Prova: Vimos no capítulo 2 que $\mathbb{E} L_{\lambda}(x, t)=S(x)+E_{x}(t)=\lambda x+\frac{t}{\lambda}$. Segue da proposição 4.1 que

$$
\mathbb{E}\left(L_{\lambda}(x, t)-\nu_{\lambda}\left(x-\frac{t}{\lambda^{2}}\right)-\frac{2 t}{\lambda}\right)^{2}=\operatorname{Var} L_{\lambda}\left(\frac{t}{\lambda^{2}}, t\right)
$$

O resultado agora segue de (4.1). 
Corolário 4.1. (Teorema Central do Limite para $L_{\lambda}$ )

Seja $\left\{\left(z_{t}, t\right): t \geq 0\right\}$ um caminho deterministico tal que $\lim _{t \rightarrow \infty} \frac{z_{t}}{t}=a$. Então

$$
\lim _{t \rightarrow \infty} \frac{\operatorname{Var}\left(L_{\lambda}\left(z_{t}, t\right)\right)}{t}=\sigma^{2}:=\left|a \lambda-\frac{1}{\lambda}\right| .
$$

Além disso, se $a \neq \lambda^{-2}$ então

$$
\lim _{t \rightarrow \infty} \mathbf{P}\left(L_{\lambda}\left(z_{t}, t\right) \leq \lambda z_{t}+\frac{t}{\lambda}+(\sigma \sqrt{t}) u\right)=\mathbf{P}(N \leq u),
$$

onde $N$ é uma variável Normal padrão.

Prova: Segue do teorema 4.1 que

$$
\lim _{t \rightarrow \infty} \frac{\mathbb{E}\left(L_{\lambda}\left(z_{t}, t\right)-\nu_{\lambda}\left(z_{t}-\frac{t}{\lambda^{2}}\right)-\frac{2 t}{\lambda}\right)^{2}}{t}=0
$$

e portanto

$$
\begin{aligned}
\lim _{t \rightarrow \infty} \frac{\operatorname{Var}\left(L_{\lambda}\left(z_{t}, t\right)\right)}{t} & =\lim _{t \rightarrow \infty} \frac{\operatorname{Var}\left(\nu_{\lambda}\left(z_{t}-\frac{t}{\lambda^{2}}\right)\right)}{t} \\
& =\left|a \lambda-\frac{1}{\lambda}\right|
\end{aligned}
$$

Como $\nu_{\lambda}$ é um processo de Poisson de taxa $\lambda$, se $a \neq \lambda^{-2}$ então $\nu_{\lambda}\left(z_{t}-\frac{t}{\lambda^{2}}\right)$ satisfaz o teorema central do limite. Junto com (4.4) isto implica (4.3).

Seja $X_{t}$ a posição no tempo t de uma partícula marcada colocada na origem de um processo de Hammersley com medida inicial invariante $\nu_{\lambda}$. Como consequência do último corolário obtemos um teorema central do limite para $X_{t}$. A partícula marcada é um conhecido processo de Markov [24] e seu comportamento é de certo modo mais simples que os das partículas de classes maiores. Nosso principal interesse aqui é introduzir o método da prova que será utilizado adiante para a partícula de segunda classe. Para tanto, utilizaremos a seguinte 
relação que deriva da definição de $L_{\lambda}(x, t)$ :

$$
L_{\lambda}(x, t)= \begin{cases}-M_{t}^{\nu_{\lambda}}\left(\left(x, X_{t}\right]\right) & \text { se } x<X_{t} \\ 0 & \text { se } x=X_{t} \\ M_{t}^{\nu_{\lambda}}\left(\left(X_{t}, x\right]\right) & \text { se } x>X_{t}\end{cases}
$$

Teorema 4.2. (Teorema Central do Limite para a partícula marcada no equilibrio)

$$
\lim _{t \rightarrow \infty} \mathbf{P}\left(X_{t} \leq-\frac{t}{\lambda^{2}}+\left(\frac{\sqrt{2 t}}{\sqrt{\lambda^{3}}}\right) u\right)=\mathbf{P}(N \leq u),
$$

onde $N$ é uma variável Normal padrão.

Prova: Segue de (4.5) que para todo $x \in \mathbb{R}$

$$
\left[X_{t} \leq x\right]=\left[L_{\lambda}(x, t) \geq 0\right]
$$

Fixe $u \in \mathbb{R}$ e defina $z_{t}:=-\frac{t}{\lambda^{2}}+\frac{\sqrt{2 t}}{\sqrt{\lambda^{3}}} u$. Para $t$ grande o suficiente, temos que $z_{t}<0$. Neste caso $L_{\lambda}\left(z_{t}, t\right)=S\left(z_{t}\right)+W(t)$. Logo,

$$
\begin{aligned}
{\left[\frac{X_{t}+\frac{t}{\lambda^{2}}}{\left.\frac{\sqrt{2 t}}{\sqrt{\lambda^{3}}} \leq u\right]}\right] } & =\left[X_{t} \leq z_{t}\right] \\
& =\left[L_{\lambda}\left(z_{t}, t\right) \geq 0\right] \\
& =\left[L_{\lambda}\left(z_{t}, t\right)-\mathbb{E} L_{\lambda}\left(z_{t}, t\right) \geq-\mathbb{E} L_{\lambda}\left(z_{t}, t\right)\right] \\
& =\left[L_{\lambda}\left(z_{t}, t\right)-\mathbb{E} S\left(z_{t}\right)-\mathbb{E} W(t) \geq-\mathbb{E} S\left(z_{t}\right)-\mathbb{E} W(t)\right] \\
& =\left[L_{\lambda}\left(z_{t}, t\right)-\frac{\sqrt{2 t}}{\sqrt{\lambda}} u \geq-\frac{\sqrt{2 t}}{\sqrt{\lambda}} u\right] \\
& =\left[-\frac{L_{\lambda}\left(z_{t}, t\right)-\frac{\sqrt{2 t}}{\sqrt{\lambda}} u}{\frac{\sqrt{2 t}}{\sqrt{\lambda}}} \leq u\right]
\end{aligned}
$$

Como $S\left(z_{t}\right)$ e $W(t)$ são processos de Poisson independentes, para $z_{t}<0$ temos que $\operatorname{Var} L_{\lambda}\left(z_{t}, t\right)=\frac{2 t}{\lambda}-\frac{\sqrt{2 t}}{\sqrt{\lambda}} u$ e pelo corolário 4.1 segue que 


$$
\lim _{t \rightarrow \infty} \frac{L_{\lambda}\left(z_{t}, t\right)-\frac{\sqrt{2 t}}{\sqrt{\lambda}} u}{\sqrt{\frac{2 t}{\lambda}-\frac{\sqrt{2 t}}{\sqrt{\lambda}} u}} \stackrel{D}{=} N
$$

em lei.

$$
\begin{gathered}
\operatorname{Como} \frac{\sqrt{\frac{2 t}{\lambda}-\frac{\sqrt{2 t}}{\sqrt{\lambda}} u}}{\frac{\sqrt{2 t}}{\sqrt{\lambda}}} \rightarrow 1 \text { quando } t \rightarrow \infty, \text { então } \\
\begin{aligned}
\lim _{t \rightarrow \infty}-\frac{L_{\lambda}\left(z_{t}, t\right)-\frac{\sqrt{2 t}}{\sqrt{\lambda}} u}{\frac{\sqrt{2 t}}{\sqrt{\lambda}}} & =\lim _{t \rightarrow \infty}-\frac{L_{\lambda}\left(z_{t}, t\right)-\frac{\sqrt{2 t}}{\sqrt{\lambda}} u}{\sqrt{\frac{2 t}{\lambda}-\frac{\sqrt{2 t}}{\sqrt{\lambda}} u}}\left(\frac{\sqrt{\frac{2 t}{\lambda}-\frac{\sqrt{2 t}}{\sqrt{\lambda}} u}}{\frac{\sqrt{2 t}}{\sqrt{\lambda}}}\right) \\
& \stackrel{D}{=}-N \\
& \stackrel{D}{=} N
\end{aligned}
\end{gathered}
$$

Portanto, segue de (4.6) e (4.7) que

$$
\lim _{t \rightarrow \infty} \mathbf{P}\left(X_{t} \leq-\frac{t}{\lambda^{2}}+\left(\frac{\sqrt{2 t}}{\sqrt{\lambda^{3}}}\right) u\right)=\mathbf{P}(N \leq u)
$$

Provaremos agora o principal resultado desta seção que diz respeito às flutuações de segunda ordem do choque microscópico $Z_{t}^{\nu_{\rho, \lambda}}$. A demonstração a seguir é uma adaptação da prova do teorema central do limite que acabamos de mostrar para a partícula de primeira classe.

Teorema 4.3. (Teorema Central do Limite para o choque)

Seja $Z_{t}^{\nu_{\rho, \lambda}}$ a posição no tempo $t$ de uma partícula de segunda classe colocada na origem de um processo de Hammersley com medida inicial no regime de choque $\nu_{\rho, \lambda}$ Poisson de taxa $\rho$ à esquerda da origem e Poisson de taxa $\lambda$ à direita da origem, com $\rho<\lambda$. Então

$$
\lim _{t \rightarrow \infty} \mathbf{P}\left(Z_{t}^{\nu_{\rho, \lambda}} \leq \frac{t}{\lambda \rho}+(\eta \sqrt{t}) u\right)=\mathbf{P}(N \leq u)
$$

onde $N$ é uma variável Normal padrão e $\eta:=\frac{\sqrt{2}}{\sqrt{\lambda \rho} \sqrt{\lambda-\rho}}$. 
Lema 4.1. Seja $Z_{t}^{\nu_{\rho, \lambda}}$ a posição no tempo t de uma partícula de segunda classe inicialmente colocada na origem de um processo de Hammersley com medida inicial $\nu_{\lambda, \rho}$, ie, Poisson de taxa $\lambda>0$ a direita da origem e Poisson de taxa $\rho>0$ a esquerda da origem, onde $\lambda>\rho$. Então, para todo $x \geq 0$

$$
\mathbf{P}\left(Z_{t}^{\nu_{\rho, \lambda}} \leq x\right)=\mathbf{P}\left(L_{\lambda}(x, t) \geq L_{\rho}(x, t)\right)
$$

Prova : Realizamos o acoplamento básico de três processos de Hammersley $\left(M_{t}^{\nu_{\rho}}\right)_{t \geq 0},\left(M_{t}^{\nu_{\lambda}}\right)_{t \geq 0}$ e $\left(M_{t}^{\nu_{\rho, \lambda}}\right)_{t \geq 0}$ de modo que as partículas de $M_{0}^{\nu_{\rho}}$ à esquerda da origem coincidam com as partículas de $M_{0}^{\nu_{\rho, \lambda}}$ à esquerda da origem e as partículas de $M_{0}^{\nu_{\lambda}}$ à direita da origem coincidam com as partículas de $M_{0}^{\nu_{\rho, \lambda}}$ à direita da origem. Do acoplamento, obtemos que $Z_{t}^{\nu_{\rho, \lambda}}=Z_{0}(t)$. Agora, (2.7) e a definição de $L_{\xi}$ implicam que

$$
\begin{aligned}
{\left[Z_{0}(t) \leq x\right] } & =\left[L_{\xi}(x, t) \geq 0\right] \\
& =\left[L_{\lambda}(x, t) \geq L_{\rho}(x, t)\right]
\end{aligned}
$$

e portanto

$$
\mathbf{P}\left(Z_{t}^{\nu_{\rho, \lambda}} \leq x\right)=\mathbf{P}\left(Z_{0}(t) \leq x\right)=\mathbf{P}\left(L_{\lambda}(x, t) \geq L_{\rho}(x, t)\right)
$$

Lema 4.2. Seja

$$
\sigma:=\frac{\sqrt{\lambda-\rho}}{\sqrt{\lambda \rho}}, \eta:=\frac{\sqrt{2}}{\sqrt{\lambda \rho} \sqrt{\lambda-\rho}} \text { e } z_{t}:=\frac{1}{\lambda \rho} t+u \eta \sqrt{t} .
$$

Defina as variáveis

$$
\Delta_{\lambda}(t):=\frac{L_{\lambda}\left(z_{t}, t\right)-\left(\lambda z_{t}+\frac{t}{\lambda}\right)}{\sigma \sqrt{t}}
$$

e

$$
\Delta_{\rho}(t):=\frac{L_{\rho}\left(z_{t}, t\right)-\left(\rho z_{t}+\frac{t}{\rho}\right)}{\sigma \sqrt{t}} .
$$

Então

$$
\left[L_{\lambda}\left(z_{t}, t\right) \geq L_{\rho}\left(z_{t}, t\right)\right]=\left[\frac{\Delta_{\rho}(t)-\Delta_{\lambda}(t)}{\sqrt{2}} \leq u\right]
$$


Prova: Usando as definições acima e fazendo as substituições obtemos:

$$
\begin{aligned}
{\left[\frac{\Delta_{\rho}(t)-\Delta_{\lambda}(t)}{\sqrt{2}} \leq u\right] } & =\left[\Delta_{\rho}(t)-\Delta_{\lambda}(t) \leq u \sqrt{2}\right] \\
& =\left[L_{\rho}\left(z_{t}, t\right)-L_{\lambda}\left(z_{t}, t\right)+(\lambda-\rho) z_{t}+\frac{t}{\lambda}-\frac{t}{\rho} \leq u \sigma \sqrt{2} \sqrt{t}\right] \\
& =\left[L_{\rho}\left(z_{t}, t\right)-L_{\lambda}\left(z_{t}, t\right)+u(\lambda-\rho) \eta \sqrt{t} \leq u \sigma \sqrt{2} \sqrt{t}\right] \\
& =\left[L_{\rho}\left(z_{t}, t\right)-L_{\lambda}\left(z_{t}, t\right)+u \sigma \sqrt{2} \sqrt{t} \leq u \sigma \sqrt{2} \sqrt{t}\right] \\
& =\left[L_{\rho}\left(z_{t}, t\right)-L_{\lambda}\left(z_{t}, t\right) \leq 0\right] \\
& =\left[L_{\lambda}\left(z_{t}, t\right) \geq L_{\rho}\left(z_{t}, t\right)\right]
\end{aligned}
$$

Prova do Teorema 4.3 Pelos lemas (4.1) e (4.2), para mostrar (4.8) é suficiente provar que $\frac{\Delta_{\rho}(t)-\Delta_{\lambda}(t)}{\sqrt{2}} \stackrel{D}{\rightarrow} N$ em lei.

Defina

$$
\begin{gathered}
A_{t}^{\rho}:=\frac{L_{\rho}\left(z_{t}, t\right)-\left[\nu_{\rho}\left(z_{t}-1 / \rho^{2}\right)+\frac{2}{\rho} t\right]}{\sigma \sqrt{2} \sqrt{t}} \\
A_{t}^{\lambda}:=\frac{L_{\lambda}\left(z_{t}, t\right)-\left[\nu_{\lambda}\left(z_{t}-1 / \lambda^{2}\right)+\frac{2}{\lambda} t\right]}{\sigma \sqrt{2} \sqrt{t}} \\
B_{t}^{\rho}:=\frac{\left[\nu_{\rho}\left(z_{t}-1 / \rho^{2}\right)+\frac{2}{\rho} t\right]-\mathbb{E}\left[\nu_{\rho}\left(z_{t}-1 / \rho^{2}\right)+\frac{2}{\rho} t\right]}{\sigma \sqrt{2} \sqrt{t}} \\
B_{t}^{\lambda}:=\frac{\left[\nu_{\lambda}\left(z_{t}-1 / \lambda^{2}\right)+\frac{2}{\lambda} t\right]-\mathbb{E}\left[\nu_{\lambda}\left(z_{t}-1 / \lambda^{2}\right)+\frac{2}{\lambda} t\right]}{\sigma \sqrt{2} \sqrt{t}}
\end{gathered}
$$


Temos que $\frac{\Delta_{\rho}(t)-\Delta_{\lambda}(t)}{\sqrt{2}}=\left(A_{t}^{\rho}-A_{t}^{\lambda}\right)-\left(B_{t}^{\rho}-B_{t}^{\lambda}\right)$.

Agora, para $t \rightarrow \infty$ segue do teorema 4.1 que

$\mathbb{E}\left[\left(A_{t}^{\rho}\right)^{2}\right] \rightarrow 0, \mathbb{E}\left[\left(A_{t}^{\lambda}\right)^{2}\right] \rightarrow 0$

Assim, $\mathbb{E}\left[\left(A_{t}^{\rho}-A_{t}^{\lambda}\right)^{2}\right] \rightarrow 0$.

Pelo teorema central do limite para o processo de Poisson temos

$\sqrt{2} B_{t}^{\rho} \stackrel{D}{\rightarrow} N$ e $\sqrt{2} B_{t}^{\lambda} \stackrel{D}{\rightarrow} N$ em lei.

Observe que para $\mathrm{t}$ grande suficiente, $z_{t}-\frac{1}{\lambda^{2}}>0$ e $z_{t}-\frac{1}{\rho^{2}}<0$, o que implica que para s e t grandes o suficiente, $B_{t}^{\rho}$ e $B_{s}^{\lambda}$ são independentes. Logo, $B_{t}^{\lambda}-B_{t}^{\rho}$ converge para $N$ em lei.

A afirmação (4.8) segue agora do teorema de Slutsky. 


\section{Capítulo 5}

\section{Coeficiente de difusão}

Neste capítulo calculamos o coeficiente de difusão do choque $\lim _{t \rightarrow \infty} \frac{\operatorname{Var} Z_{t}^{\nu \rho, \lambda}}{t}$ para o processo de Hammersley. Nossa prova se baseia na conhecida relação entre o fluxo de partículas de segunda classe $L_{\xi}$ e a posição da partícula marcada de segunda classe no processo de duas classes $Z_{0}(t)$. Obtemos essa relação de dois modos distintos: o primeiro pode ser utilizado para obter relações análogas em outros processos totalmente assimétricos e o segundo se extende para o caso do processo de Hammmersley parcialmente assimétrico.

A seguinte proposição relaciona fluxo com a posição da partícula marcada e pode ser adaptada para uma classe grande de processos totalmente assimétricos com conservação de massa. Esta relação é útil para calcular de maneira simples os momentos de uma partícula marcada e também pode ser utilizada para partículas marcadas de classes maiores.

Considere um processo de Hammersley com medida inicial $\mu$. Seja $X_{t}^{a}$ a posição no tempo $t$ de uma partícula marcada cuja posição inicial $a$ é um ponto de $\mu$. Defina a variável $\chi_{t}^{\mu}(x):=\sup \left\{a \in \mathbb{R}: X_{t}^{a} \leq x, \mu(\{a\})=1\right\}$. Temos:

Proposição 5.1. Seja $\left(M_{t}^{\nu^{\prime}}\right)_{t \geq 0}$ um processo de Hammersley clássico com medida inicial $\nu^{\prime}$ igual a $\nu$ fora da origem e acrescida de uma partícula na origem, cuja posição no tempo $t$ denotaremos por $X_{t}^{0}$. Então,

$$
L_{\nu^{\prime}}(x, t)=\nu^{\prime}\left(\chi_{t}^{\nu^{\prime}}(x)\right)
$$

e para quaisquer $f$ continua e $y<0$

$$
\mathbb{E}\left[f\left(\nu^{\prime}\left(\chi_{t}^{\nu^{\prime}}(x)\right)\right) \mid \chi_{t}^{\nu^{\prime}}(x)=y\right]=\mathbb{E}\left[f\left(\nu^{\prime}(y)\right)\right]
$$


Além disso, se $\nu$ é invariante por translaçôes, temos

$$
\begin{array}{cll}
\chi_{t}^{\nu^{\prime}}(x) & \stackrel{D}{=} & x-X_{t}^{0} \\
\left(\chi_{t}^{\nu^{\prime}}(x)_{-}\right. & \stackrel{D}{=} & \left.\left(x-X_{t}^{0}\right)_{-}\right)
\end{array}
$$

em lei.

Prova :

Como o sistema se mantém ordenado, temos que

$$
\nu^{\prime}\left(\chi_{t}^{\nu^{\prime}}(x)\right)= \begin{cases}-M_{t}^{\nu^{\prime}}\left(\left(x, X_{t}^{0}\right]\right) & \text { se } x<X_{t}^{0} \\ 0 & \text { se } x=X_{t}^{0} \\ M_{t}^{\nu^{\prime}}\left(\left(X_{t}^{0}, x\right]\right) & \text { se } x>X_{t}^{0}\end{cases}
$$

Junto com (4.5) obtemos

$$
L_{\nu^{\prime}}(x, t)=\nu^{\prime}\left(\chi_{t}^{\nu^{\prime}}(x)\right)
$$

Agora, seja f contínua e $y<0$, a equação

$$
\mathbb{E}\left[f\left(\nu^{\prime}\left(\chi_{t}^{\nu^{\prime}}(x)\right)\right) \mid \chi_{t}^{\nu^{\prime}}(x)=y\right]=\mathbb{E}\left[f\left(\nu^{\prime}(y)\right)\right]
$$

segue da definição de $\chi_{t}^{\nu^{\prime}}(x)$ e do fato da trajetória de uma partícula marcada qualquer $\left(X_{t}^{a}\right)_{t \geq 0}$ depender apenas da configuração à sua esquerda (pois os saltos são da direita para a esquerda). Observe que dada uma realização onde $\chi_{t}^{\nu^{\prime}}(x)=y<0$, então se fixarmos $\mathbf{P}$ e $\nu((-\infty, y])$ mas modificarmos $\nu((y,+\infty))$, temos que $\chi_{t}^{\nu^{\prime}}(x)$ não se altera.

Por fim, observe que $\left[X_{t}^{0} \leq x\right]$ se e somente se $\left[\chi_{t}^{\nu^{\prime}}(x) \geq 0\right]$. Assim,

$$
\begin{aligned}
{\left[x-X_{t}^{0} \geq h\right] } & =\left[-X_{t}^{0} \geq h-x\right] \\
& =\left[X_{t}^{0} \leq x-h\right] \\
& =\left[\chi_{t}^{\nu^{\prime}}(x-h) \geq 0\right]
\end{aligned}
$$

Agora, se transladarmos a origem de 0 para $-h$ e denotarmos por $\pi_{-h}(\nu)^{\prime}$ a nova medida inicial ( $\nu$ transladada por $-h$ e acrescida de uma partícula na 
nova origem), então

$$
\left[\chi_{t}^{\nu^{\prime}}(x-h) \geq 0\right]=\left[\chi_{t}^{\pi_{-h}(\nu)^{\prime}}(x-h) \geq h\right]
$$

Pela invariância por translações da $\nu$ e da dinâmica, segue que $\chi_{t}^{\pi_{-h}(\nu)^{\prime}}(x-$ $h) \stackrel{D}{=} \chi_{t}^{\nu^{\prime}}(x)$ em lei. Logo,

$$
\begin{aligned}
x-X_{t}^{0} & =\chi_{t}^{\pi_{-h}(\nu)^{\prime}}(x-h) \\
& \stackrel{D}{=} \chi_{t}^{\nu^{\prime}}(x)
\end{aligned}
$$

em lei.

Como aplicação da proposição 5.1 calcularemos a variância da partícula marcada e a esperança do choque no processo de Hammersley. Começamos com a partícula marcada.

Teorema 5.1. Seja $X_{t}$ a posição no tempo $t$ da partícula marcada colocada na origem no tempo 0 em um processo de Hammersley em equilibrio com medida inicial $\nu$ Poisson de intensidade $\lambda$. Então para todo $t \geq 0$,

$$
\mathbb{E} X_{t}=-\frac{t}{\lambda^{2}}
$$

$$
\operatorname{Var} X_{t}=\frac{2 t}{\lambda^{3}}
$$

Prova: Começaremos com a esperança. Segue da proposição 5.1 que

$$
\begin{aligned}
\mathbb{E}\left(L_{\lambda}(x, t)_{-}\right) & =\mathbb{E}\left(\nu\left(\chi_{t}(x)_{-}\right)\right) \\
& =\mathbb{E}\left[\mathbb{E}\left(\nu\left(\chi_{t}(x)_{-}\right) \mid \chi_{t}(x)_{-}\right)\right] \\
& =\mathbb{E}\left[\lambda \chi_{t}(x)_{-}\right] \\
& =\lambda \mathbb{E}\left[\left(x-X_{t}\right)_{-}\right]
\end{aligned}
$$

Agora observe que $\left|L_{\lambda}(x, t)_{-}-L_{\lambda}(x, t)\right|=\left|L_{\lambda}(x, t)_{+}\right| \leq\left|L_{\lambda}(x, t)\right|$ e que $L_{\lambda}(x, t)_{+}$decresce a 0 quase certamente quando $x$ decresce para menos infinito. 
Assim, pelo teorema da convergência dominada

$$
\lim _{x \rightarrow-\infty} \mathbb{E}\left(L_{\lambda}(x, t)_{-}\right)-\mathbb{E}\left(L_{\lambda}(x, t)\right)=0
$$

Como para todo $y \in \mathbb{R}$ temos $\lim _{x \rightarrow-\infty} \mathbf{1}_{\left[X_{t}>x\right]}(y)=1$ quase certamente, então também segue pelo teorema da convergência dominada que

$$
\lim _{x \rightarrow-\infty} \mathbb{E}\left[\left(x-X_{t}\right)_{-}\right]-\mathbb{E}\left[x-X_{t}\right]=0
$$

o que implica

$$
\lim _{x \rightarrow-\infty} \lambda \mathbb{E}\left[x-X_{t}\right]-\mathbb{E}\left(L_{\lambda}(x, t)\right)=0
$$

Como para $x<0$

$$
\begin{aligned}
\mathbb{E}\left(L_{\lambda}(x, t)\right) & =\mathbb{E}(S(x)+W(t)) \\
& =\lambda x+\frac{t}{\lambda}
\end{aligned}
$$

segue que

$$
\lim _{x \rightarrow-\infty} \lambda x-\lambda \mathbb{E}\left[X_{t}\right]-\lambda x-\frac{t}{\lambda}=0
$$

e portanto

$$
\mathbb{E}\left(X_{t}\right)=-\frac{t}{\lambda^{2}}
$$

Da mesma maneira podemos calcular o segundo momento.

Da proposição 5.1 segue que

$$
\begin{aligned}
\mathbb{E}\left(L_{\lambda}(x, t)_{-}^{2}\right) & =\mathbb{E}\left(\nu\left(\chi_{t}(x)_{-}\right)^{2}\right) \\
& =\mathbb{E}\left[\mathbb{E}\left(\nu\left(\chi_{t}(x)_{-}\right)^{2} \mid \chi_{t}(x)_{-}\right)\right] \\
& =\mathbb{E}\left[\lambda^{2} \chi_{t}(x)_{-}^{2}+\lambda \chi_{t}(x)_{-}\right] \\
& =\lambda^{2} \mathbb{E}\left[\left[\left(x-X_{t}\right)_{-}\right]^{2}\right]+\lambda \mathbb{E}\left[\left(x-X_{t}\right)_{-}\right]
\end{aligned}
$$

Agora observe que $\left|L_{\lambda}(x, t)_{-}^{2}-L_{\lambda}(x, t)^{2}\right| \leq\left(L_{\lambda}(x, t)_{-}-L_{\lambda}(x, t)\right)^{2}=L_{\lambda}(x, t)_{+}^{2}$ e que $L_{\lambda}(x, t)_{+}$decresce a 0 quase certamente quando $x$ decresce para menos infinito. Assim, pelo teorema da convergência dominada

$$
\lim _{x \rightarrow-\infty} \mathbb{E}\left(L_{\lambda}(x, t)_{-}^{2}\right)-\mathbb{E}\left(L_{\lambda}(x, t)^{2}\right)=0
$$

Como para todo $y \in \mathbb{R}$ temos $\lim _{x \rightarrow-\infty} \mathbf{1}_{\left[X_{t}>x\right]}(y)=1$ quase certamente, então 
também segue pelo teorema da convergência dominada que

$$
\lim _{x \rightarrow-\infty} \mathbb{E}\left[\left[\left(x-X_{t}\right)_{-}\right]^{2}\right]-\mathbb{E}\left[\left(x-X_{t}\right)^{2}\right]=0
$$

o que implica

$$
\lim _{x \rightarrow-\infty} \lambda^{2} \mathbb{E}\left[\left(x-X_{t}\right)^{2}\right]+\lambda \mathbb{E}\left[x-X_{t}\right]-\mathbb{E}\left(L_{\lambda}(x, t)^{2}\right)=0
$$

Para $x<0$ e $t \geq 0, L_{\lambda}(x, t)=S(x)+W(t)$. Como $S(x)$ e $W(t)$ são independentes, temos

$$
\begin{aligned}
\mathbb{E}\left(L_{\lambda}(x, t)^{2}\right) & =\mathbb{E}\left(S(x)^{2}+2 S(x) W(t)+W(t)^{2}\right) \\
& =\mathbb{E}\left(S(x)^{2}\right)+2 \mathbb{E}(S(x)) \mathbb{E}(W(t))+\mathbb{E}\left(W(t)^{2}\right) \\
& =\lambda^{2} x^{2}+\lambda x+2 \lambda x \frac{t}{\lambda}+\frac{t^{2}}{\lambda^{2}}+\frac{t}{\lambda} \\
& =\lambda^{2} x^{2}+\lambda x+2 x t+\frac{t^{2}}{\lambda^{2}}+\frac{t}{\lambda}
\end{aligned}
$$

segue que

$$
\begin{aligned}
0 & =\lim _{x \rightarrow-\infty} \lambda^{2} \mathbb{E}\left[\left(x-X_{t}\right)^{2}\right]+\lambda \mathbb{E}\left[x-X_{t}\right]-\mathbb{E}\left(L_{\lambda}(x, t)^{2}\right) \\
& =\lim _{x \rightarrow-\infty} \lambda^{2} \mathbb{E}\left[\left(x-X_{t}\right)^{2}\right]+\mathbb{E}\left[L_{\lambda}(x, t)\right]-\mathbb{E}\left(L_{\lambda}(x, t)^{2}\right) \\
& =\lim _{x \rightarrow-\infty} \lambda^{2} x^{2}+2 \lambda^{2} x \frac{t}{\lambda^{2}}+\lambda^{2} \mathbb{E}\left[X_{t}^{2}\right]+\lambda x+\frac{t}{\lambda}-\lambda^{2} x^{2}-\lambda x-2 x t-\frac{t^{2}}{\lambda^{2}}-\frac{t}{\lambda} \\
& =\lambda^{2} \mathbb{E}\left[X_{t}^{2}\right]-\frac{2 t}{\lambda}-\frac{t^{2}}{\lambda^{2}}
\end{aligned}
$$

e portanto

$$
\mathbb{E}\left(X_{t}^{2}\right)=\frac{2 t}{\lambda^{3}}+\frac{t^{2}}{\lambda^{4}}
$$

Subtraindo o quadrado da esperança, temos que

$$
\operatorname{Var} X_{t}=\frac{2 t}{\lambda^{3}}
$$

A proposição 5.1 também vale para partículas marcadas de segunda classe no processo de Hammersley multiclasse. Para tanto, considere um processo de Hammersley de duas classes com medida inicial $\left(\nu_{\lambda}, \nu_{\xi}\right)$ e defina $Z_{t}^{a}$ a posição no tempo $t$ de uma partícula marcada de segunda classe cuja posição inicial $a$ é 
um ponto de $\mu_{\xi}$. Defina a variável $\zeta_{t}^{\mu_{\xi}}(x):=\sup \left\{a \in \mathbb{R}: Z_{t}^{a} \leq x, \mu_{\xi}(\{a\})=1\right\}$.

Proposição 5.2. Defina $\nu_{\lambda}:=\nu_{\rho}+\nu_{\xi}$ onde $\nu_{\rho}$ e $\nu_{\xi}$ são dois processos de Poisson independentes em $\mathbb{R}$ com taxas $\rho$ e $(\lambda-\rho)$ respectivamente. Acrescente uma partícula na origem à $\nu_{\xi}$ (e consequentemente à $\nu_{\lambda}$ ). Denote essas novas medidas por $\nu_{\xi}^{\prime}$ e $\nu_{\lambda}^{\prime} O$ acoplamento $\left(M_{t}^{\nu_{\rho}}, M_{t}^{\nu_{\lambda}^{\prime}}\right)_{t \geq 0}$ define um sistema de choque onde $Z_{t}^{\nu_{\rho, \lambda}}=Z_{0}(t)$. Temos que

$$
L_{\xi}(x, t)=\nu_{\xi}^{\prime}\left(\zeta_{t}^{\nu_{\xi}^{\prime}}(x)\right)
$$

e para quaisquer $f$ contínua e $y \geq 0$

$$
\mathbb{E}\left[f\left(\nu_{\xi}^{\prime}\left(\zeta_{t}^{\nu_{\xi}^{\prime}}(x)\right)\right) \mid \zeta_{t}^{\nu_{\xi}^{\prime}}(x)=y\right]=\mathbb{E}\left[f\left(\nu_{\xi}^{\prime}(y)\right)\right]
$$

Além disso,

$$
\begin{array}{ccl}
\zeta_{t}^{\nu_{\xi}^{\prime}}(x) & \stackrel{D}{=} & x-Z_{t}^{0} \\
\left(\zeta_{t}^{\nu_{\xi}^{\prime}}(x)_{+}\right. & \stackrel{D}{=} & \left.\left(x-Z_{t}^{0}\right)_{+}\right)
\end{array}
$$

em lei.

Omitiremos a prova por ser igual à da proposição 5.1. Como antes, podemos utilizar esta relação para estudar os momentos de $Z_{t}^{\nu_{\rho, \lambda}}$. Por exemplo, temos

Proposição 5.3. Seja $Z_{t}^{\nu_{\rho, \lambda}}$ a posição no tempo $t$ de uma partícula de segunda classe isolada colocada na origem no tempo 0 em um processo de Hammersley em equilíbrio com medida inicial de choque $\nu_{\rho, \lambda}$, Poisson de intensidade $\lambda$ à direita da origem e Poisson de intensidade $\rho$ à esquerda da origem, com $\lambda>\rho>0$. Então para todo $t \geq 0$,

$$
\mathbb{E} Z_{t}^{\nu_{\rho, \lambda}}=\frac{t}{\lambda \rho}
$$

Prova :

Segue da proposição 5.2 que 


$$
\begin{aligned}
\mathbb{E}\left(L_{\xi}(x, t)_{+}\right) & =\mathbb{E}\left(\nu_{\xi}\left(\zeta_{t}(x)_{+}\right)\right) \\
& =\mathbb{E}\left[\mathbb{E}\left(\nu_{\xi}\left(\zeta_{t}(x)_{+}\right) \mid \zeta_{t}(x)_{+}\right)\right] \\
& =\mathbb{E}\left[(\lambda-\rho) \zeta_{t}(x)_{+}\right] \\
& =(\lambda-\rho) \mathbb{E}\left[\left(x-Z_{t}^{\nu_{\rho, \lambda}}\right)_{+}\right]
\end{aligned}
$$

Agora observe que $\left|L_{\xi}(x, t)_{+}-L_{\xi}(x, t)\right|=\left|L_{\xi}(x, t)_{-}\right| \leq\left|L_{\xi}(x, t)\right|$ e que $L_{\xi}(x, t)$ _ decresce a 0 quase certamente quando $x$ cresce para infinito. Assim, pelo teorema da convergência dominada

$$
\lim _{x \rightarrow \infty} \mathbb{E}\left(L_{\xi}(x, t)_{+}\right)-\mathbb{E}\left(L_{\xi}(x, t)\right)=0
$$

Como para todo $y \in \mathbb{R}$ temos $\lim _{x \rightarrow \infty} \mathbf{1}_{\left[Z_{t}^{\nu \rho, \lambda} \leq x\right]}(y)=1$ quase certamente, então também segue pelo teorema da convergência dominada que

$$
\lim _{x \rightarrow \infty} \mathbb{E}\left[\left(x-Z_{t}^{\nu_{\rho, \lambda}}\right)_{+}\right]-\mathbb{E}\left[x-Z_{t}^{\nu_{\rho, \lambda}}\right]=0
$$

o que implica

$$
\lim _{x \rightarrow \infty}(\lambda-\rho) \mathbb{E}\left[x-Z_{t}^{\nu_{\rho, \lambda}}\right]-\mathbb{E}\left(L_{\xi}(x, t)\right)=0
$$

Como

$$
\begin{aligned}
\mathbb{E}\left(L_{\xi}(x, t)\right) & =\mathbb{E}\left(L_{\lambda}(x, t)-L_{\rho}(x, t)\right) \\
& =\mathbb{E}\left(S_{\lambda}(x)-E_{\lambda}(t)\right)-\mathbb{E}\left(S_{\rho}(x)-E_{\rho}(t)\right) \\
& =(\lambda-\rho)\left(x-\frac{t}{\lambda \rho}\right)
\end{aligned}
$$

segue que

$$
\lim _{x \rightarrow \infty}(\lambda-\rho) x-(\lambda-\rho) \mathbb{E}\left[Z_{t}^{\nu \rho, \lambda}\right]-(\lambda-\rho)\left(x-\frac{t}{\lambda \rho}\right)=0
$$

e portanto

$$
\mathbb{E}\left(Z_{t}^{\nu_{\rho, \lambda}}\right)=\frac{t}{\lambda \rho}
$$

A variância do fluxo de partículas de segunda classe não é tão simples de 
se obter como a variância do fluxo de primeira classe. O próximo teorema nos dá uma boa aproximação. Para o caso da medida invariante multiclasse, mostraremos uma fórmula exata no capítulo 6 .

Teorema 5.2. Seja $\left\{\left(z_{t}, t\right): t \geq 0\right\}$ um caminho deterministico, então

$$
\begin{aligned}
0 & <\liminf _{t \rightarrow \infty} \frac{\mathbb{E}\left(L_{\xi}\left(z_{t}, t\right)-\left[\nu_{\lambda}\left(z_{t}-\frac{t}{\lambda^{2}}\right)+\frac{2 t}{\lambda}-\nu_{\rho}\left(z_{t}-\frac{t}{\rho^{2}}\right)-\frac{2 t}{\rho}\right]\right)^{2}}{t^{\frac{2}{3}}} \\
& \leq \limsup _{t \rightarrow \infty} \frac{\mathbb{E}\left(L_{\xi}\left(z_{t}, t\right)-\left[\nu_{\lambda}\left(z_{t}-\frac{t}{\lambda^{2}}\right)+\frac{2 t}{\lambda}-\nu_{\rho}\left(z_{t}-\frac{t}{\rho^{2}}\right)-\frac{2 t}{\rho}\right]\right)^{2}}{t^{\frac{2}{3}}}<\infty
\end{aligned}
$$

\section{Prova do Teorema 5.2.}

Defina

$$
m:=\liminf _{t \rightarrow \infty} \frac{\operatorname{Var} L_{1}(t, t)}{t^{\frac{2}{3}}}
$$

e

$$
M:=\limsup _{t \rightarrow \infty} \frac{\operatorname{Var} L_{1}(t, t)}{t^{\frac{2}{3}}}
$$

Sabemos pela proposição 4.1 que para qualquer $\gamma>0, L_{\gamma}\left(z_{t}, t\right)-\nu_{\gamma}\left(z_{t}-\right.$ $\left.\frac{t}{\gamma^{2}}\right) \stackrel{D}{=} L_{\gamma}\left(\frac{t}{\gamma^{2}}, t\right)$ em lei. Como a aplicação $(x, t) \mapsto\left(\gamma x, \frac{t}{\gamma}\right)$ transforma um processo de Hammersley de taxa $\gamma$ em um processo de Hammersley de taxa 1 temos que $L_{\gamma}(x, t) \stackrel{D}{=} L_{1}\left(\gamma x, \frac{t}{\gamma}\right)$ em lei. Assim,

$$
L_{\gamma}\left(z_{t}, t\right)-\nu_{\gamma}\left(z_{t}-\frac{t}{\gamma^{2}}\right) \stackrel{D}{=} L_{\gamma}\left(\frac{t}{\gamma^{2}}, t\right) \stackrel{D}{=} L_{1}\left(\frac{t}{\gamma}, \frac{t}{\gamma}\right)
$$

em lei 
Como

$$
\begin{aligned}
\liminf _{t \rightarrow \infty} \frac{\mathbb{E}\left(L_{\gamma}\left(z_{t}, t\right)-\nu_{\gamma}\left(z_{t}-\frac{t}{\gamma^{2}}\right)-\frac{2 t}{\gamma}\right)^{2}}{t^{\frac{2}{3}}} & =\liminf _{t \rightarrow \infty} \frac{1}{\gamma^{\frac{2}{3}}} \frac{\operatorname{Var} L_{1}\left(\frac{t}{\gamma}, \frac{t}{\gamma}\right)}{\left(\frac{t}{\gamma}\right)^{\frac{2}{3}}} \\
& =\frac{m}{\gamma^{\frac{2}{3}}}
\end{aligned}
$$

e

$$
\begin{aligned}
\limsup _{t \rightarrow \infty} \frac{\mathbb{E}\left(L_{\gamma}\left(z_{t}, t\right)-\nu_{\gamma}\left(z_{t}-\frac{t}{\gamma^{2}}\right)-\frac{2 t}{\gamma}\right)^{2}}{t^{\frac{2}{3}}} & =\limsup _{t \rightarrow \infty} \frac{1}{\gamma^{\frac{2}{3}}} \frac{\operatorname{Var} L_{1}\left(\frac{t}{\gamma}, \frac{t}{\gamma}\right)}{\left(\frac{t}{\gamma}\right)^{\frac{2}{3}}} \\
& =\frac{M}{\gamma^{\frac{2}{3}}}
\end{aligned}
$$

Defina

$$
\begin{aligned}
& A_{t}^{\rho}:=L_{\rho}\left(z_{t}, t\right)-\nu_{\rho}\left(z_{t}-\frac{t}{\rho^{2}}\right)-\frac{2 t}{\rho} \\
& A_{t}^{\lambda}:=L_{\lambda}\left(z_{t}, t\right)-\nu_{\lambda}\left(z_{t}-\frac{t}{\lambda^{2}}\right)-\frac{2 t}{\lambda}
\end{aligned}
$$


Segue da desigualdade triangular e do teorema 4.1 que

$$
\begin{aligned}
0 & <\left(\frac{1}{\lambda^{\frac{1}{3}}}-\frac{1}{\rho^{\frac{1}{3}}}\right)^{2} m \\
& =\liminf _{t \rightarrow \infty} \frac{\left(\left[\mathbb{E}\left(A_{t}^{\lambda}\right)^{2}\right]^{\frac{1}{2}}-\left[\mathbb{E}\left(A_{t}^{\rho}\right)^{2}\right]^{\frac{1}{2}}\right)^{2}}{t^{\frac{2}{3}}} \\
& \leq \liminf _{t \rightarrow \infty} \frac{\mathbb{E}\left(L_{\xi}\left(z_{t}, t\right)-\left[\nu_{\lambda}\left(z_{t}-\frac{t}{\lambda^{2}}\right)+\frac{2 t}{\lambda}-\nu_{\rho}\left(z_{t}-\frac{t}{\rho^{2}}\right)-\frac{2 t}{\rho}\right]\right)^{2}}{t^{\frac{2}{3}}} \\
& \leq \limsup _{t \rightarrow \infty} \frac{\mathbb{E}\left(L_{\xi}\left(z_{t}, t\right)-\left[\nu_{\lambda}\left(z_{t}-\frac{t}{\lambda^{2}}\right)+\frac{2 t}{\lambda}-\nu_{\rho}\left(z_{t}-\frac{t}{\rho^{2}}\right)-\frac{2 t}{\rho}\right]\right)^{2}}{t^{\frac{2}{3}}} \\
& \leq \limsup _{t \rightarrow \infty} \frac{\left(\left[\mathbb{E}\left(A_{t}^{\lambda}\right)^{2}\right]^{\frac{1}{2}}+\left[\mathbb{E}\left(A_{t}^{\rho}\right)^{2}\right]^{\frac{1}{2}}\right)^{2}}{t^{\frac{2}{3}}} \\
& =\left(\frac{1}{\lambda^{\frac{1}{3}}}+\frac{1}{\rho^{\frac{1}{3}}}\right)^{2} M<\infty
\end{aligned}
$$

Utilizando o teorema acima e a proposição 5.2 podemos proceder de modo análogo ao utilizado para a partícula de primeira classe para obtermos o coeficiente de difusão do choque. Entretanto, se quisermos calcular o coeficiente de difusão do choque para processos em que a partícula de segunda classe salta para os dois lados como no caso do ASEP, a proposição 5.2 não seria mais válida pois o processo de partículas de segunda classe não é totalmente assimétrico neste caso. Para processos parcialmente assimétricos, se a medida inicial for Poisson ou produto, podemos utilizar uma técnica introduzida por Ferrari e Fontes [14] para o ASEP e adaptada para o processo de Hammersley por Coletti, Ferrari e Pimentel [12] para relacionar os momentos do fluxo com os momentos da partícula marcada. A seguir provaremos uma versão da prova de Coletti, Ferrari e Pimentel [12] para o caso (parcialmente) assimétrico.

Seja $\lambda>\rho>0$. Defina $\nu_{\lambda}:=\nu_{\rho}+\nu_{\xi}$ onde $\nu_{\rho}$ e $\nu_{\xi}$ são dois processos de Poisson independentes em $\mathbb{R}$ com taxas $\rho$ e $(\lambda-\rho)$ respectivamente. Para essa medida inicial o fluxo de partículas de segunda classe satisfaz o seguinte: 
Lema 5.1. Para todo $x \in \mathbb{R}$ et $\geq 0$,

$$
\mathbb{E} L_{\xi}(x, t)_{+}=(\lambda-\rho) \mathbb{E}\left(x-Z_{t}^{\nu \rho, \lambda}\right)_{+}
$$

e

$$
\mathbb{E} L_{\xi}(x, t)_{-}=(\lambda-\rho) \mathbb{E}\left(Z_{t}^{\nu_{\rho, \lambda}}-x\right)_{+}
$$

Prova do Lema 5.1. segue da definição que

$$
L_{\xi}(x, t)_{+}=\int_{0}^{\infty} \mathbf{1}\left\{Z_{y}(t) \leq x\right\} \nu_{\xi}(d y)
$$

em que $Z_{y}(t)$ denota a posição no tempo $t$ da partícula de segunda classe que inicialmente se encontra em $y \in[0, \infty]$. Pelo teorema de Slyvnjak-Mecke e pela invariância por translações da medida inicial temos

$$
\begin{aligned}
\mathbb{E} L_{\xi}(x, t)_{+} & =(\lambda-\rho) \int_{0}^{\infty} \mathbb{P}\left(Z_{y}(t) \leq x\right) d y \\
& =(\lambda-\rho) \int_{0}^{\infty} \mathbb{P}\left(Z_{t}^{\nu_{\rho, \lambda}} \leq x-y\right) d y \\
& =(\lambda-\rho) \int_{0}^{\infty} \mathbb{P}\left(Z_{t}^{\nu_{\rho, \lambda}}-x \leq-y\right) d y \\
& =(\lambda-\rho) \int_{0}^{\infty} \mathbb{P}\left(x-Z_{t}^{\nu_{\rho, \lambda}} \geq y\right) d y \\
& =(\lambda-\rho) \mathbb{E}\left(x-Z_{t}^{\nu_{\rho, \lambda}}\right)_{+}
\end{aligned}
$$

Analogamente

$$
\begin{aligned}
\mathbb{E} L_{\xi}(x, t)_{-} & =(\lambda-\rho) \int_{-\infty}^{0} \mathbb{P}\left(Z_{y}(t)>x\right) d y \\
& =(\lambda-\rho) \int_{-\infty}^{0} \mathbb{P}\left(Z_{t}^{\nu_{\rho, \lambda}}>x-y\right) d y \\
& =(\lambda-\rho) \int_{0}^{\infty} \mathbb{P}\left(Z_{t}^{\nu_{\rho, \lambda}}-x>z\right) d z \\
& =(\lambda-\rho) \mathbb{E}\left(Z_{t}^{\nu_{\rho, \lambda}}-x\right)_{+}
\end{aligned}
$$


Uma idéia análoga funciona para calcularmos $\operatorname{Var}\left(Z_{t}^{\nu_{\rho, \lambda}}\right)$. Para o caso totalmente assimétrico, temos:

Proposição 5.4. (Coletti, Ferrari e Pimentel [12])

Para todo $x, t \geq 0$

$$
\begin{aligned}
\operatorname{Var} L_{\xi}(x, t)_{+}= & (\lambda-\rho)\left(x-\int_{0}^{x} \mathbb{P}\left(Z_{t}^{\nu_{\rho, \lambda}}>z\right) d z\right) \\
& +(\lambda-\rho)^{2}\left(\int_{0}^{x} 2 z \mathbb{P}\left(Z_{t}^{\nu_{\rho, \lambda}}>z\right) d z-\left(\int_{0}^{x} \mathbb{P}\left(Z_{t}^{\nu_{\rho, \lambda}}>z\right) d z\right)^{2}\right)
\end{aligned}
$$

Provaremos o caso assimétrico a seguir.

Proposição 5.5. Para todo $x \in \mathbb{R}$ et $\geq 0$

$$
\operatorname{Var} L_{\xi}(x, t)_{+}=(\lambda-\rho) \mathbb{E}\left(x-Z_{t}^{\nu_{\rho, \lambda}}\right)_{+}+(\lambda-\rho)^{2} \operatorname{Var}\left(x-Z_{t}^{\nu_{\rho, \lambda}}\right)_{+}
$$

\section{Prova da Proposição 5.5}

Seja $Z_{y}(t)$ a posição no tempo $t$ da partícula de segunda classe que inicialmente se encontra em $y \in[0, \infty]$. Observe que se $\bar{y} \leq y$ e $Z_{y}(t) \leq x$ então $Z_{\bar{y}}(t) \leq x$. Assim, do mesmo modo que fazemos com o quadrado da soma de funções com valores em $\{0,1\}$, temos

$$
L_{\xi}(x, t)_{+}^{2}=L_{\xi}(x, t)_{+}+2 \int_{0}^{\infty} \int_{0}^{y} \mathbf{1}\left\{Z_{y}(t) \leq x\right\} \nu_{\xi}(d \bar{y}) \nu_{\xi}(d y)
$$

Pelo teorema de Slyvnjak-Mecke e pela invariância por translações da medida inicial, a esperança da integral dupla na equação acima é dada por

$$
\begin{aligned}
\mathbb{E}\left(2 \int_{0}^{\infty} \int_{0}^{y} \mathbf{1}\left\{Z_{y}(t) \leq x\right\} \nu_{\xi}(d \bar{y}) \nu_{\xi}(d y)\right)=(\lambda-\rho)^{2} \int_{0}^{\infty} \int_{0}^{y} 2 \mathbb{P}\left(Z_{y}(t) \leq x\right) d \bar{y} d y \\
=(\lambda-\rho)^{2} \int_{0}^{\infty} \int_{0}^{y} 2 \mathbb{P}\left(Z_{t}^{\nu_{\rho, \lambda}} \leq x-y\right) d \bar{y} d y \\
=(\lambda-\rho)^{2} \int_{0}^{\infty} 2 y \mathbb{P}\left(Z_{t}^{\nu_{\rho, \lambda}} \leq x-y\right) d y \\
=(\lambda-\rho)^{2} \int_{0}^{\infty} 2 y \mathbb{P}\left(x-Z_{t}^{\nu_{\rho, \lambda}} \geq y\right) d y
\end{aligned}
$$


Portanto pelo lema 5.1, temos

$$
\begin{aligned}
\operatorname{Var} L_{\xi}(x, t)_{+}= & \mathbb{E}\left(\left[L_{\xi}(x, t)_{+}\right]^{2}\right)-\left(\mathbb{E} L_{\xi}(x, t)_{+}\right)^{2} \\
= & \mathbb{E}\left(L_{\xi}(x, t)_{+}\right)+(\lambda-\rho)^{2} \int_{0}^{\infty} 2 y \mathbb{P}\left(x-Z_{t}^{\nu_{\rho, \lambda}} \geq y\right) d y \\
& -\left(\mathbb{E} L_{\xi}(x, t)_{+}\right)^{2} \\
= & (\lambda-\rho) \mathbb{E}\left(x-Z_{t}^{\nu_{\rho, \lambda}}\right)_{+} \\
& +(\lambda-\rho)^{2}\left[\int_{0}^{\infty} 2 y \mathbb{P}\left(x-Z_{t}^{\nu_{\rho, \lambda}} \geq y\right) d y-\mathbb{E}\left[\left(x-Z_{t}^{\nu_{\rho, \lambda}}\right)_{+}\right]^{2}\right] \\
= & (\lambda-\rho) \mathbb{E}\left(x-Z_{t}^{\nu_{\rho, \lambda}}\right)_{+}+(\lambda-\rho)^{2} \operatorname{Var}\left(x-Z_{t}^{\nu_{\rho, \lambda}}\right)_{+}
\end{aligned}
$$

Deste resultado obtemos o seguinte teorema para o choque $Z_{t}^{\nu_{\rho, \lambda}}$ no processo de Hammersley clássico. Sua extensão para o caso assimétrico depende da prova que a medida invariante pra esse caso também é Poisson.

Teorema 5.3. Seja $Z_{t}^{\nu_{\rho, \lambda}}$ a posição no tempo $t$ de uma partícula de segunda classe colocada na origem de um processo de Hammersley com medida inicial no regime de choque $\nu_{\rho, \lambda}$, Poisson de taxa $\rho$ à esquerda da origem e Poisson de taxa $\lambda$ à direita da origem, $\operatorname{com} \rho<\lambda$. Para todo $t \geq 0$ e $\epsilon>0$,

$$
\operatorname{Var} Z_{t}^{\nu_{\rho, \lambda}}=\frac{2 t}{\lambda \rho(\lambda-\rho)}+o\left(t^{\epsilon+2 / 3}\right)
$$

\section{Prova do Teorema 5.3.}

Seja $\epsilon>0$ e $x=c t, \operatorname{com} c \geq \frac{1}{\rho^{2}}$. Como consequência do teorema 5.2 temos que

$$
\begin{aligned}
\operatorname{Var} L_{\xi}(x, t) & =\operatorname{Var}\left(\nu_{\lambda}\left(x-\frac{t}{\lambda^{2}}\right)-\nu_{\rho}\left(x-\frac{t}{\rho^{2}}\right)\right)+o\left(t^{\epsilon+2 / 3}\right) \\
& =\operatorname{Var}\left(\nu_{\xi}\left(x-\frac{t}{\lambda^{2}}\right)\right)+\operatorname{Var}\left(\nu_{\rho}\left(x-\frac{t}{\rho^{2}}, x-\frac{t}{\lambda^{2}}\right)\right)+o\left(t^{\epsilon+2 / 3}\right) \\
& =(\lambda-\rho)\left(x-\frac{t}{\rho^{2}}\right)+\lambda t\left(\frac{1}{\rho^{2}}-\frac{1}{\lambda^{2}}\right)+o\left(t^{\epsilon+2 / 3}\right) \\
& =(\lambda-\rho)\left(x+\frac{t}{\lambda \rho}\right)+o\left(t^{\epsilon+2 / 3}\right)
\end{aligned}
$$


Por outro lado

$\operatorname{Var} L_{\xi}(x, t)=\operatorname{Var} L_{\xi}(x, t)_{+}+\operatorname{Var} L_{\xi}(x, t)_{-}+2 \mathbb{E}\left(L_{\xi}(x, t)_{+}\right) \mathbb{E}\left(L_{\xi}(x, t)_{-}\right)$

Portanto por (5.10) e pela proposição 5.4

$$
\begin{aligned}
&(\lambda-\rho)\left(x+\frac{t}{\lambda \rho}\right)+o\left(t^{\epsilon+2 / 3}\right)= \\
&= \operatorname{Var} L_{\xi}(x, t)_{+}+\operatorname{Var} L_{\xi}(x, t)_{-}+2 \mathbb{E}\left(L_{\xi}(x, t)_{+}\right) \mathbb{E}\left(L_{\xi}(x, t)_{-}\right) \\
&=(\lambda-\rho)\left(x-\int_{0}^{x} \mathbb{P}\left(Z_{t}^{\nu_{\rho, \lambda}}>z\right) d z\right) \\
& \quad+(\lambda-\rho)^{2}\left(\int_{0}^{x} 2 z \mathbb{P}\left(Z_{t}^{\nu_{\rho, \lambda}}>z\right) d z-\left(\int_{0}^{x} \mathbb{P}\left(Z_{t}^{\nu_{\rho, \lambda}}>z\right) d z\right)^{2}\right) \\
& \quad+\operatorname{Var} L_{\xi}(x, t)_{-}+2 \mathbb{E}\left(L_{\xi}(x, t)_{+}\right) \mathbb{E}\left(L_{\xi}(x, t)_{-}\right)
\end{aligned}
$$

o que implica

$$
\begin{array}{r}
(\lambda-\rho)^{2}\left(\int_{0}^{x} 2 z \mathbb{P}\left(Z_{t}^{\nu_{\rho, \lambda}}>z\right) d z-\left(\int_{0}^{x} \mathbb{P}\left(Z_{t}^{\nu_{\rho, \lambda}}>z\right) d z\right)^{2}\right)= \\
=(\lambda-\rho)\left(\frac{t}{\lambda \rho}+\int_{0}^{x} \mathbb{P}\left(Z_{t}^{\nu_{\rho, \lambda}}>z\right) d z\right)-o\left(t^{\epsilon+2 / 3}\right) \\
\quad-\operatorname{Var} L_{\xi}(x, t)_{-}-2 \mathbb{E}\left(L_{\xi}(x, t)_{+}\right) \mathbb{E}\left(L_{\xi}(x, t)_{-}\right)
\end{array}
$$

Agora observe que $\left|L_{\xi}(x, t)_{-}\right| \leq\left|L_{\xi}(x, t)\right|$ e que $L_{\xi}(x, t)_{-}$decresce a 0 quase certamente quando $x$ cresce para infinito. Assim, pelo teorema da convergência dominada

$$
\lim _{x \rightarrow \infty} \operatorname{Var} L_{\xi}(x, t)_{-}=0
$$

Provaremos agora que $\lim _{x \rightarrow \infty} \mathbb{E}\left(L_{\xi}(x, t)_{+}\right) \mathbb{E}\left(L_{\xi}(x, t)_{-}\right)=0$. 
Pelo lema 5.1 é suficiente mostrar que $\lim _{x \rightarrow \infty} \int_{0}^{x} \mathbb{P}\left(Z_{t}^{\nu_{\rho, \lambda}} \leq z\right) d z \int_{x}^{\infty} \mathbb{P}\left(Z_{t}^{\nu_{\rho, \lambda}}>\right.$ $z) d z=0$.

Temos que

$$
\begin{array}{rl}
\lim _{x \rightarrow \infty} \int_{0}^{x} & \mathbb{P}\left(Z_{t}^{\nu_{\rho, \lambda}} \leq z\right) d z \int_{x}^{\infty} \mathbb{P}\left(Z_{t}^{\nu_{\rho, \lambda}}>z\right) d z= \\
= & \lim _{x \rightarrow \infty} \int_{0}^{x} 1-\mathbb{P}\left(Z_{t}^{\nu_{\rho, \lambda}}>z\right) d z \int_{x}^{\infty} \mathbb{P}\left(Z_{t}^{\nu_{\rho, \lambda}}>z\right) d z \\
= & \lim _{x \rightarrow \infty} \int_{x}^{\infty} x \mathbb{P}\left(Z_{t}^{\nu_{\rho, \lambda}}>z\right) d z \\
& \quad-\lim _{x \rightarrow \infty} \int_{0}^{x} \mathbb{P}\left(Z_{t}^{\nu_{\rho, \lambda}}>z\right) d z \int_{x}^{\infty} \mathbb{P}\left(Z_{t}^{\nu_{\rho, \lambda}}>z\right) d z \\
\leq & \lim _{x \rightarrow \infty} \int_{x}^{\infty} z \mathbb{P}\left(Z_{t}^{\nu_{\rho, \lambda}}>z\right) d z \\
& \quad-\lim _{x \rightarrow \infty} \int_{0}^{x} \mathbb{P}\left(Z_{t}^{\nu_{\rho, \lambda}}>z\right) d z \int_{x}^{\infty} \mathbb{P}\left(Z_{t}^{\nu_{\rho, \lambda}}>z\right) d z \\
= & \lim _{x \rightarrow \infty} \int_{x}^{\infty} z \mathbb{P}\left(Z_{t}^{\nu_{\rho, \lambda}}>z\right) d z-\mathbb{E}\left(Z_{t}^{\nu_{\rho, \lambda}}\right) \lim _{x \rightarrow \infty} \int_{x}^{\infty} \mathbb{P}\left(Z_{t}^{\nu_{\rho, \lambda}}>z\right) d z \\
= & 0
\end{array}
$$

onde os dois últimos limites vão a zero pelo teorema da convergência dominada mais a integrabilidade de $\left(Z_{t}^{\nu_{\rho, \lambda}}\right)^{2}$ e $Z_{t}^{\nu_{\rho, \lambda}}$.

Por fim, fazendo $\mathrm{x}$ tender a infinito nos dois lados de (5.11) segue que

$$
(\lambda-\rho)^{2} \operatorname{Var} Z_{t}^{\nu_{\rho, \lambda}}=2(\lambda-\rho) \frac{t}{\lambda \rho}+o\left(t^{\epsilon+2 / 3}\right)
$$

e temos o resultado.

Como corolário imediato obtemos o coeficiente de difusão do choque $D$ : 
Corolário 5.1. Seja $D=\lim _{t \rightarrow \infty} \frac{\operatorname{Var} Z_{t}^{\nu \rho, \lambda}}{t}$. Temos que

$$
D=\frac{2}{\lambda \rho(\lambda-\rho)}
$$




\section{Capítulo 6}

\section{Variância do Fluxo no Equilíbrio}

Dado $\lambda>0$, definimos $Z^{\nu_{\lambda}}(t)$ como sendo a posição no tempo t de uma partícula isolada de segunda classe colocada na origem de um processo de Hammersley com medida inicial invariante Poisson de intensidade $\rho$. Neste capítulo mostraremos uma fórmula que relaciona a variância do fluxo de partículas de segunda classe $L_{\xi}(x, t)$ numa versão estacionária do processo Hammersley multiclasse com a localização da partícula isolada de segunda classe $Z^{\nu_{1}}(t)$ de um processo de Hammersley clássico com medida inicial invariante Poisson de taxa 1. Esta fórmula deriva de uma fórmula análoga obtida por Cator e Groeneboom [9] para a o fluxo de partículas de primeira classe $L_{\lambda}$ no processo de Hammersley clássico em equilíbrio que enunciamos abaixo:

Teorema 6.1. (Variância do fluxo de primeira classe $L_{\lambda}$ )

Sejam $x$ e $t \in \mathbb{R}^{+}$, temos que

$$
\operatorname{Var} L_{\lambda}(x, t)=-\lambda x+\frac{t}{\lambda}+2 \lambda \mathbb{E}\left(x-Z^{\nu_{\lambda}}(t)\right)_{+}
$$

Junto com a Lei dos grandes números para $Z^{\nu_{\lambda}}(t)$, esta fórmula implica a sublinearidade da variância do fluxo na direção característica $\mathbb{E} Z^{\nu_{\lambda}}(t)=\frac{t}{\lambda^{2}}$. Além disso, ela foi crucial para a obtenção da ordem correta da variância do fluxo em [9]. A seguir calculamos a esperança da posição da partícula isolada de segunda classe para todo tempo $t$ introduzindo o método utilizado em [9] para provar o teorema 6.1. A mesma técnica também será usada para o fluxo de segunda classe $L_{\xi}$. Temos:

Proposição 6.1. Seja $Z^{\nu_{\lambda}}(t)$ a posição no tempo $t$ de uma partícula de segunda classe isolada inicialmente posta na origem de um processo de Hammersley es- 
tacionário de taxa $\lambda$. Para todo $t \in \mathbb{R}$,

$$
\mathbb{E} Z^{\nu_{\lambda}}(t)=\frac{t}{\lambda^{2}}
$$

\section{Prova da Proposição}

Vamos fazer um contagem dupla na variância do fluxo de partículas de primeira classe, $\operatorname{Var}\left(L_{\lambda}\right)$, a partir da identidade $L_{\lambda}=N+W=S+E$. Começamos calculando $\operatorname{Var}(N+W)$ para $t>0$, como em Cator e Groeneboom [9].

$$
\begin{aligned}
\operatorname{Var}(N+W) & =\operatorname{Cov}(N+W, N+W) \\
& =\operatorname{Var}(W)+\operatorname{Var}(N)+2 \operatorname{Cov}(N, W) \\
& =\operatorname{Var}(W)+\operatorname{Var}(N)+2 \operatorname{Cov}(N, S+E-N) \\
& =\operatorname{Var}(W)-\operatorname{Var}(N)+2 \operatorname{Cov}(S, N) \\
& =\frac{t}{\lambda}-\lambda x+2 \operatorname{Cov}(S, N)
\end{aligned}
$$

Queremos encontrar uma relação entre a covariância acima e a esperança de $Z^{\nu_{\lambda}}(t)$. Para tanto, vamos variar o processo $S$ para uma taxa $\lambda+\gamma \operatorname{com} \gamma \geq 0$. Mantemos $W$ com a mesma intensidade. Denotamos por $\mathbb{E}_{\gamma}$ as esperanças com relação a essa nova taxa para $S$ e $a_{n}:=\mathbb{E}_{\gamma}(N \mid S=n)$. Observe que como a distribuição dos pontos de $\mathrm{S}$ é uniforme dado $S$, segue que $a_{n}$ não depende de $\gamma$. Assim,

$$
\begin{aligned}
\left.\partial \gamma\right|_{\gamma=0} \mathbb{E}_{\gamma}(N) & =\left.\partial \gamma\right|_{\gamma=0} \sum_{n=0}^{\infty} \frac{((\lambda+\gamma) t)^{n}}{n !} e^{-t(\lambda+\gamma)} a_{n} \\
& =\frac{1}{\lambda}\left(\sum_{n=0}^{\infty} \frac{(\lambda t)^{n}}{n !} e^{-t \lambda} a_{n} n\right)-t \sum_{n=0}^{\infty} \frac{(\lambda t)^{n}}{n !} e^{-t \lambda} a_{n} \\
& =\frac{1}{\lambda} \mathbb{E}(N S)-t \mathbb{E}(N)
\end{aligned}
$$

Logo,

$$
\operatorname{Cov}(N, S)=\left.\lambda \partial \gamma\right|_{\gamma=0} \mathbb{E}_{\gamma}(N)
$$

Agora, denotando por $N^{\gamma}$ o norte do processo com a taxa da fonte variada, percebemos que para $\gamma$ suficientemente pequeno $N^{\gamma}-N=1$ se, e somente, se $Z_{y}(t) \leq x$, onde $Z_{y}(t)$ denota a posição no tempo t de uma partícula de segunda 
classe inicialmente posta no ponto y. Assim, como $\gamma$ é um processo de Poisson, temos

$$
\mathbb{E}\left(N^{\gamma}-N\right)=\gamma \int_{0}^{x} \mathbb{E}\left(\mathbf{1}_{Z_{y}(t) \leq x}\right) d y+O\left(\gamma^{2}\right)
$$

Como o processo visto da partícula de segunda classe isolada é um processo de Poisson invariante por traslações, segue que $Z_{y}$ tem a mesma distribuição para todo y. Temos então que

$$
\begin{aligned}
\operatorname{Cov}(N, S) & =\left.\lambda \partial \gamma\right|_{\gamma=0} \mathbb{E}_{\gamma}(N) \\
& =\lambda \int_{0}^{x} \mathbb{E}\left(\mathbf{1}_{Z_{y}(t) \leq x}\right) d y \\
& =\lambda \int_{0}^{x} \mathbb{P}\left(Z_{0}(t) \leq x-y\right) d y \\
& =\lambda \int_{0}^{x} \mathbb{P}\left(Z_{0}(t) \leq y\right) d y \\
& =\lambda \int_{0}^{x} \mathbb{P}\left(Z^{\nu_{\lambda}}(t) \leq y\right) d y
\end{aligned}
$$

Assim,

$$
\operatorname{Var}(N+W)=\frac{t}{\lambda}-\lambda x+2 \lambda \int_{0}^{x} \mathbb{P}\left(Z^{\nu_{\lambda}}(t) \leq y\right) d y
$$

Agora, observe que se rotacionarmos o processo em $\theta=\frac{\pi}{2}$ radianos no sentido anti-horário obtemos um processo de Hammersley com as partículas saltando no sentido oposto, ie, da esquerda para a direita. Assim, denotando os processos rotacionados por $S_{\theta}, W_{\theta}, N_{\theta}$ e $E_{\theta}$ temos que $S_{\theta}=W, W_{\theta}=S, N_{\theta}=E$, $E_{\theta}=N$. A partícula de segunda classe no processo rotacionado denotamos por $Z_{\theta}$ e observamos que $\left[Z_{\theta}(x) \leq t\right]=\left[Z^{\nu_{\lambda}}(t)>x\right]$. Procedendo de modo análogo, 
temos:

$$
\begin{aligned}
\operatorname{Var}\left(L_{\lambda}\right) & =\operatorname{Var}(S+E) \\
& =\operatorname{Var}\left(W_{\theta}+N_{\theta}\right) \\
& =\operatorname{Var}\left(W_{\theta}\right)-\operatorname{Var}\left(N_{\theta}\right)+\frac{2}{\lambda} \int_{0}^{t} \mathbb{P}\left(Z_{\theta}(x) \leq y\right) d y \\
& =\operatorname{Var}(S)-\operatorname{Var}(E)+\frac{2}{\lambda} \int_{0}^{t} \mathbb{P}\left(Z^{\nu_{\lambda}}(y)>x\right) d y \\
& =\lambda x-\frac{t}{\lambda}+\frac{2}{\lambda} \int_{0}^{t} \mathbb{P}\left(Z^{\nu_{\lambda}}(y)>x\right) d y
\end{aligned}
$$

Portanto,

$$
\begin{aligned}
& \frac{t}{\lambda}-\lambda x+2 \lambda \int_{0}^{x} \mathbb{P}\left(Z^{\nu_{\lambda}}(t) \leq y\right) d y=\lambda x-\frac{t}{\lambda}+\frac{2}{\lambda} \int_{0}^{t} \mathbb{P}\left(Z^{\nu_{\lambda}}(u)>x\right) d u \\
& \Leftrightarrow \\
& \frac{t}{\lambda}-\lambda x+2 \lambda \int_{0}^{x} 1-\mathbb{P}\left(Z^{\nu_{\lambda}}(t)>y\right) d y=\lambda x-\frac{t}{\lambda}+\frac{2}{\lambda} \int_{0}^{t} 1-\mathbb{P}\left(Z^{\nu_{\lambda}}(u) \leq x\right) d u \\
& \Leftrightarrow \quad \lambda \int_{0}^{x} \mathbb{P}\left(Z^{\nu_{\lambda}}(t)>y\right) d y=\frac{1}{\lambda} \int_{0}^{t} \mathbb{P}\left(Z^{\nu_{\lambda}}(u) \leq x\right) d u
\end{aligned}
$$

Assim, vemos que $\forall t \geq 0$

$$
\begin{aligned}
\mathbb{E}\left(Z^{\nu_{\lambda}}(t)\right) & =\lim _{x \rightarrow \infty} \int_{0}^{x} \mathbb{P}\left(Z^{\nu_{\lambda}}(t)>y\right) d y \\
& =\lim _{x \rightarrow \infty} \frac{1}{\lambda^{2}} \int_{0}^{t} \mathbb{P}\left(Z^{\nu_{\lambda}}(u) \leq x\right) d u \\
& =\frac{t}{\lambda^{2}}
\end{aligned}
$$

Para $t<0$ o resultado segue da reversibilidade do Hammersley.

Agora mostraremos o principal resultado deste capítulo. Para tanto, construímos o processo de Hammersley com duas classes $\left(M_{t}^{\nu \rho}, \xi_{t}\right)_{t \geq 0}$ a partir de um 
processo multilinha $\left(M_{t}^{\alpha_{1}}, M_{t}^{\alpha_{1}}, \mathbf{P}\right), \operatorname{com}\left(M_{t}^{\nu_{\rho}}, \xi_{t}\right)_{t \geq 0}=\Phi\left(\Psi\left(\left(M_{t}^{\alpha_{1}}, M_{t}^{\alpha_{2}}, \mathbf{P}\right)\right)\right)$ (ver seção sobre o choque, capítulo 3 , para mais detalhes). Vimos que para cada tempo t, a medida invariante multiclasse pode ser representada pelo processo de saída de uma fila $M / M / 1$ gerada pelos processos de chegada $M_{t}^{\alpha_{1}}$ e serviços $M_{t}^{\alpha_{2}}$. Uma observação importante para as identidades a seguir, é que nessa fila o tempo x caminha de $+\infty$ para $-\infty$.

Abaixo seguem algumas relações importantes utilizadas na prova que podem ser derivadas desta representação. Defina:

$Q_{0}(t):=$ número de clientes na fila (do tempo t) no instante 0 $Q_{x}(t):=$ número de clientes na fila (do tempo t) no instante $\mathrm{x}$

$N_{\rho}:=$ número de clientes atendidos no intervalo $(0, \mathrm{x})$ na fila do tempo $\mathrm{t}$

$$
\begin{aligned}
& =Q_{x}(t)+N_{\alpha_{1}}-Q_{0}(t) \\
& =N_{\alpha_{1}}-\left(Q_{0}(t)-Q_{x}(t)\right)
\end{aligned}
$$

$N_{\xi}:=$ número de serviços não utilizados no intervalo $(0, \mathrm{x})$ na fila do tempo $\mathrm{t}$ $=N_{\lambda}-N_{\rho}$$$
=N_{\alpha_{2}}-\left(N_{\alpha_{1}}-\left(Q_{0}(t)-Q_{x}(t)\right)\right)
$$$$
\left.=N_{\alpha_{2}}-N_{\alpha_{1}}+\left(Q_{0}(t)-Q_{x}(t)\right)\right)
$$

Por fim, dizemos que $f(x, t)=O(1)$ se $\exists M \in \mathbb{R}$ tal que $|f(x, t)|<M$ para todo $(x, t) \in \mathbb{R}^{2}$.

Vale o seguinte resultado:

Proposição 6.2. Seja $L_{\xi}(x, t)$ o fluxo de partículas de segunda classe da origem a $(x, t)$ em um processo de duas classes com medida inicial invariante com densidades $\lambda$ e $\lambda-\rho$ de partículas de primeira e segunda classe, respectivamente, 
onde $\lambda>\rho$. Sejam $x, t \in \mathbb{R}^{+}$, temos que

$$
\begin{aligned}
& \operatorname{Var} L_{\xi}(x, t)=(\lambda+\rho)\left(\frac{t}{\lambda \rho}-x\right) \\
& \quad+2(\lambda x-t / \lambda) \mathbb{P}\left(Z^{\nu_{1}}(t / \lambda) \leq \lambda x\right)+2(\rho x-t / \rho) \mathbb{P}\left(Z^{\nu_{1}}(t / \rho) \leq \rho x\right) \\
& \quad+2 \mathbb{E}\left(\left[t / \lambda-Z^{\nu_{1}}(t / \lambda)\right] \mathbf{1}_{\left[Z^{\left.\nu_{1}(t / \lambda) \leq \lambda x\right]}\right)+2 \mathbb{E}\left(\left[t / \rho-Z^{\nu_{1}}(t / \rho)\right] \mathbf{1}_{\left[Z^{\nu_{1}}(t / \rho) \leq \rho x\right]}\right)}\right. \\
& \quad+O(1)
\end{aligned}
$$

\section{Prova da Proposição}

Como $x$ e $t$ estão fixados, para clarear a notação omitiremos eles abaixo sempre que possível. Temos que

$$
\begin{aligned}
\operatorname{Var} L_{\xi}(x, t) & =\operatorname{Cov}\left(N_{\xi}+W_{\xi}, N_{\xi}+W_{\xi}\right) \\
& =\operatorname{Var}\left(N_{\xi}\right)+\operatorname{Var}\left(W_{\xi}\right)+2 \operatorname{Cov}\left(N_{\xi}, W_{\xi}\right)
\end{aligned}
$$

Calcularemos cada um desses termos.

Primeiro, pela invarância da medida inicial

$$
\begin{aligned}
& \operatorname{Var}\left(N_{\xi}\right)=\operatorname{Var}\left(S_{\xi}\right)= \\
& \quad=\operatorname{Var}\left(S_{\alpha_{2}}-S_{\alpha_{1}}+\left(Q_{0}-Q_{x}\right)\right) \\
& \quad=\operatorname{Var}\left(S_{\alpha_{2}}-S_{\alpha_{1}}\right)+\operatorname{Var}\left(Q_{0}-Q_{x}\right)+2 \operatorname{Cov}\left(S_{\alpha_{2}}-S_{\alpha_{1}}, Q_{0}-Q_{x}\right)
\end{aligned}
$$

Observe que tanto $Q_{x}$ como $Q_{0}$ têm Lei igual ao do número de clientes numa fila $M / M / 1$ em equilíbrio, que é uma variável geométrica de parâmetro $\frac{\rho}{\lambda}<1$. Portanto, para qualquer $x$ constante, $Q_{x}$ e $Q_{0}$ possuem os mesmos momentos finitos (e independentes de t). Assim,

$$
\begin{aligned}
\operatorname{Var}\left(Q_{0}-Q_{x}\right) & =\operatorname{Var}\left(Q_{0}\right)+\operatorname{Var}\left(Q_{x}\right)-2 \operatorname{Cov}\left(Q_{0}, Q_{x}\right) \\
& =2 \operatorname{Var}\left(Q_{0}\right)-2 \operatorname{Cov}\left(Q_{0}, Q_{x}\right) \\
& \leq 4 \operatorname{Var}\left(Q_{0}\right)
\end{aligned}
$$

Da onde

$$
\operatorname{Var}\left(Q_{0}-Q_{x}\right)=O(1)
$$


Agora,

$$
\begin{aligned}
& \operatorname{Cov}\left(S_{\alpha_{2}}-S_{\alpha_{1}}, Q_{0}-Q_{x}\right)= \\
& \quad=\operatorname{Cov}\left(S_{\alpha_{2}}, Q_{0}\right)+\operatorname{Cov}\left(S_{\alpha_{1}}, Q_{x}\right)-\operatorname{Cov}\left(S_{\alpha_{2}}, Q_{x}\right)-\operatorname{Cov}\left(S_{\alpha_{1}}, Q_{0}\right) \\
& \quad=\operatorname{Cov}\left(S_{\alpha_{2}}, Q_{0}\right)-\operatorname{Cov}\left(S_{\alpha_{1}}, Q_{0}\right)
\end{aligned}
$$

pois as chegadas e os serviços futuros $S_{\alpha_{1}}$ e $S_{\alpha_{2}}$ não dependem do número de clientes na fila no presente $Q_{x}$.

Considere agora o processo reverso da fila $\left(Q_{y}\right)_{y \geq 0}$ onde o tempo y é crescente. Este processo também é uma fila $M / M / 1$ cujo processo de chegada de clientes é $S_{\rho}$ e o processo de serviços é $S_{\alpha_{1}}+S_{\xi}$. Como antes, estes processos não dependem do número de clientes na fila reversa no presente $Q_{0}$ (Teorema de Burke) . Logo

$$
\operatorname{Cov}\left(S_{\rho}, Q_{0}\right)=0
$$

e

$$
\operatorname{Cov}\left(S_{\alpha_{1}}+S_{\xi}, Q_{0}\right)=0
$$

Como $S_{\rho}=S_{\alpha_{1}}-Q_{0}+Q_{x}$ e $S_{\xi}=S_{\alpha_{2}}-S_{\alpha_{1}}+Q_{0}-Q_{x}$, segue das equações acima que

$$
\operatorname{Cov}\left(S_{\alpha_{1}}, Q_{0}\right)=\operatorname{Cov}\left(Q_{0}-Q_{x}, Q_{0}\right)=-\operatorname{Cov}\left(S_{\alpha_{2}}, Q_{0}\right)
$$

Portanto,

$$
\begin{aligned}
2 \operatorname{Cov}\left(S_{\alpha_{2}}-S_{\alpha_{1}}, Q_{0}-Q_{x}\right) & =2 \operatorname{Cov}\left(S_{\alpha_{2}}, Q_{0}\right)-2 \operatorname{Cov}\left(S_{\alpha_{1}}, Q_{0}\right) \\
& =4 \operatorname{Cov}\left(Q_{x}-Q_{0}, Q_{0}\right) \\
& =4 \operatorname{Cov}\left(Q_{0}, Q_{x}\right)-4 \operatorname{Var}\left(Q_{0}\right) \\
& =-2 \operatorname{Var}\left(Q_{0}-Q_{x}\right)
\end{aligned}
$$

Como $S_{\alpha_{1}}$ e $S_{\alpha_{2}}$ são processos de Poisson independentes, segue que

$$
\begin{aligned}
\operatorname{Var}\left(N_{\xi}\right) & =\operatorname{Var}\left(S_{\xi}\right) \\
& =\operatorname{Var}\left(S_{\alpha_{2}}-S_{\alpha_{1}}\right)+\operatorname{Var}\left(Q_{0}-Q_{x}\right)+2 \operatorname{Cov}\left(S_{\alpha_{2}}-S_{\alpha_{1}}, Q_{0}-Q_{x}\right) \\
& =\operatorname{Var}\left(S_{\alpha_{2}}\right)+\operatorname{Var}\left(S_{\alpha_{1}}\right)-\operatorname{Var}\left(Q_{0}-Q_{x}\right) \\
& =(\lambda+\rho) x+O(1)
\end{aligned}
$$


Utilizando o corolário 3.1, obtemos relações análogas para $W_{\xi}$. Procedendo como antes, temos que

$$
\operatorname{Var}\left(W_{\xi}\right)=\left(\frac{1}{\rho}+\frac{1}{\lambda}\right) t+O(1)
$$

Nos resta calcular a $\operatorname{Cov}\left(N_{\xi}, W_{\xi}\right)$. Vale:

$$
\begin{aligned}
\operatorname{Cov}\left(N_{\xi}, W_{\xi}\right) & =\operatorname{Cov}\left(N_{\xi}, S_{\xi}+E_{\xi}-N_{\xi}\right) \\
& =\operatorname{Cov}\left(N_{\xi}, S_{\xi}\right)+\operatorname{Cov}\left(N_{\xi}, E_{\xi}\right)-\operatorname{Var}\left(N_{\xi}\right) \\
& =\operatorname{Cov}\left(N_{\xi}, S_{\xi}\right)+\operatorname{Cov}\left(S_{\xi}, W_{\xi}\right)-\operatorname{Var}\left(N_{\xi}\right)
\end{aligned}
$$

Vamos justificar esta última igualdade. Temos que $\operatorname{Var}\left(N_{\xi}+W_{\xi}\right)=\operatorname{Var}\left(S_{\xi}+E_{\xi}\right)$. Como $N_{\xi}$ tem a mesma lei de $S_{\xi}$ e $E_{\xi}$ tem a mesma lei de $W_{\xi}$, segue que $\operatorname{Cov}\left(N_{\xi}, E_{\xi}\right)=\operatorname{Cov}\left(S_{\xi}, W_{\xi}\right)$.

Agora, sejam $Z^{\nu_{\lambda}}(t)$ e $Z^{\nu_{\rho}}(t)$ as posições de duas partículas de segunda classe isoladas colocadas na origem de duas cópias independentes dos processos $\left(M_{t}^{\nu \lambda}\right)_{t \geq 0}$ e $\left(M_{t}^{\nu_{\rho}}\right)_{t \geq 0}$ respectivamente. Temos,

$$
\begin{aligned}
\operatorname{Cov}\left(N_{\xi}, S_{\xi}\right) & =\operatorname{Cov}\left(N_{\lambda}-N_{\rho}, S_{\lambda}-S_{\rho}\right) \\
& =\operatorname{Cov}\left(N_{\lambda}, S_{\lambda}\right)+\operatorname{Cov}\left(N_{\rho}, S_{\rho}\right)-\operatorname{Cov}\left(N_{\lambda}, S_{\rho}\right)-\operatorname{Cov}\left(N_{\rho}, S_{\lambda}\right)
\end{aligned}
$$

Os dois primeiros termos são conhecidos, valem $\lambda \mathbb{E}\left(x-Z^{\nu_{\lambda}}(t)\right)_{+}$e $\rho \mathbb{E}(x-$ $\left.Z^{\nu_{\rho}}(t)\right)_{+}$respectivamente (ver Cator e Groeneboom [9]). Vamos investigar a $\operatorname{Cov}\left(N_{\rho}, S_{\lambda}\right)$. Como feito na proposição 6.1 , acrescentamos ao processo $S_{\lambda}$ um processo de Poisson de taxa $\gamma$ independente dos outros processos. Mantemos estes outros processos inalterados. Desta maneira obtemos um novo $N_{\rho}$, o qual denotaremos por $N_{\rho}^{\gamma}$, e como antes temos

$$
\operatorname{Cov}\left(N_{\rho}, S_{\lambda}\right)=\left.\lambda \partial \gamma\right|_{\gamma=0} \mathbb{E}\left(N_{\rho}^{\gamma}\right)=\lambda \lim _{\gamma \rightarrow 0} \frac{\mathbb{E}\left(N_{\rho}^{\gamma}-N_{\rho}\right)}{\gamma}
$$

Quando $\gamma$ é suficientemente pequeno temos que $S_{\lambda}^{\gamma}-S_{\lambda} \leq 1$ a menos de um erro de ordem $\gamma^{2}$. Considere a fila $M / M / 1$ obtida pelo processo multilinha que gera o processo $\left(M_{t}^{\nu_{\lambda}}, M_{t}^{\nu \rho}\right)_{t \geq 0}$. Se esta nova partícula cai num período de 
desocupação da fila (intervalo de tempo em que a fila permanece vazia), então o processo $\nu_{\rho}$ permanece inalterado e $N_{\rho}^{\gamma}-N_{\rho}=0$. Porém, se a nova partícula cai em um período de ocupação da fila surgem duas discrepâncias entre $S_{\rho}^{\gamma}$ e $S_{\rho}$. A primeira na posição da nova partícula. Denotamos a posição no tempo t desta partícula de segunda classe que inicialmente está num ponto y por $Z_{y}(t)$. Além disso, todas as partículas de $S_{\rho}$ a esquerda de $Z_{y}(0)$ e que estão dentro do mesmo período de ocupação avançam (no espaço) para a posição onde estava a partícula imediatamente à sua direita. Como resultado, a última partícula deste periodo de ocupação (primeira com relação à origem) se tranforma numa partícula de segunda classe e, assim, não pertence a $S_{\rho}^{\gamma}$. Isto gera a outra discrepância, cuja posição no tempo t denotaremos por $\bar{Z}_{\bar{y}}(t)$, onde $\bar{y}<y$ é sua posição inicial. Observe que $y-\bar{y}=O(1)$ (não depende de $\mathrm{t}$, por exemplo). Acoplando o processo $\left(M_{t}^{\nu_{\rho}}\right)$ com o processo modificado e observando que $\bar{y}$ é função determinística de y e da fila, em resumo, obtemos

$$
\mathbb{E}\left(N_{\rho}^{\gamma}-N_{\rho}\right)=\gamma \mathbb{P}\left(Q_{y}>0\right) \mathbb{E}\left(\int_{0}^{x} \mathbf{1}_{\left[Z_{y}(t)>x\right]} \mathbf{1}_{\left[\bar{Z}_{\bar{y}}(t) \leq x\right]} d y\right)+O\left(\gamma^{2}\right)
$$

Logo,

$$
\begin{aligned}
\operatorname{Cov}\left(N_{\rho}, S_{\lambda}\right) & =\lambda \lim _{\gamma \rightarrow 0} \frac{\mathbb{E}\left(N_{\rho}^{\gamma}-N_{\rho}\right)}{\gamma} \\
& =\lambda \mathbb{P}\left(Q_{y}>0\right) \mathbb{E}\left(\int_{0}^{x} \mathbf{1}_{\left[Z_{y}(t)>x\right]} \mathbf{1}_{\left[\bar{Z}_{\bar{y}}(t) \leq x\right]} d y\right) \\
& =\lambda \rho \int_{0}^{x} \mathbb{P}\left(Z_{y}(t)>x, \bar{Z}_{\bar{y}}(t) \leq x\right) d y
\end{aligned}
$$

como $Z_{y}(t) \geq \bar{Z}_{\bar{y}}(t)$ para todo t, então

$$
1=\mathbb{P}\left(Z_{y}(t)>x, \bar{Z}_{\bar{y}}(t) \leq x\right)+\mathbb{P}\left(Z_{y}(t) \leq x\right)+\mathbb{P}\left(\bar{Z}_{\bar{y}}(t)>x\right)
$$


Junto com a invariância por translação do processo implica

$$
\begin{aligned}
\mathbb{P}\left(Z_{y}(t)>x, \bar{Z}_{\bar{y}}(t) \leq x\right) & =1-\mathbb{P}\left(Z_{y}(t) \leq x\right)-\mathbb{P}\left(\bar{Z}_{\bar{y}}(t)>x\right) \\
& =1-\mathbb{P}\left(Z_{0}(t) \leq x-y\right)-\mathbb{P}\left(\bar{Z}_{0}(t)>x-\bar{y}\right) \\
& =1-\mathbb{P}\left(x-Z_{0}(t) \geq y\right)-\mathbb{P}\left(x-\bar{Z}_{0}(t)<\bar{y}\right) \\
& =\mathbb{P}\left(x-\bar{Z}_{0}(t)>\bar{y}\right)-\mathbb{P}\left(x-Z_{0}(t) \geq y\right) \\
& =\mathbb{P}\left(x-Z^{\nu_{\rho}}(t)>\bar{y}\right)-\mathbb{P}\left(x-Z^{\nu_{\rho}}(t)>y\right) \\
& =\mathbb{P}\left(x-Z^{\nu_{\rho}}(t)+O(1)>y\right)-\mathbb{P}\left(x-Z^{\nu_{\rho}}(t)>y\right)
\end{aligned}
$$

Portanto

$$
\begin{aligned}
\operatorname{Cov} & \left(N_{\rho}, S_{\lambda}\right)=\lambda \rho \int_{0}^{x} \mathbb{P}\left(Z_{y}(t)>x, \bar{Z}_{\bar{y}}(t) \leq x\right) d y \\
& =\lambda \rho \int_{0}^{x} \mathbb{P}\left(x-Z^{\nu_{\rho}}(t)+O(1)>y\right)-\mathbb{P}\left(x-Z^{\nu_{\rho}}(t)>y\right) d y \\
& =\lambda \rho \mathbb{E}\left(\left[x-Z^{\nu_{\rho}}(t)\right] \mathbf{1}_{\left[Z^{\nu_{\rho}}(t) \leq x\right]}-\left[x-Z^{\nu_{\rho}}(t)+O(1)\right] \mathbf{1}_{\left[Z^{\nu \rho}(t) \leq x+O(1)\right]}\right) \\
& =\lambda \rho \mathbb{E}\left(O(1) \mathbf{1}_{\left[Z^{\nu \rho}(t) \leq x\right]}-\left[x-Z^{\nu_{\rho}}(t)\right] \mathbf{1}_{\left[x<Z^{\nu \rho}(t) \leq x+O(1)\right]}\right) \\
& =\lambda \rho\left[O(1) \mathbb{P}\left(Z^{\nu_{\rho}}(t) \leq x\right)+\mathbb{E}\left(\left[Z^{\nu_{\rho}}(t)-x\right] \mathbf{1}_{\left[0 \leq Z^{\nu \rho}(t)-x<O(1)\right]}\right)\right] \\
& =O(1)
\end{aligned}
$$

Agora, vale que

$$
\begin{aligned}
\operatorname{Cov}\left(N_{\lambda}, S_{\rho}\right) & =\operatorname{Cov}\left(N_{\alpha_{2}}, S_{\alpha_{1}}-\left(Q_{0}-Q_{x}\right)\right) \\
& =\operatorname{Cov}\left(N_{\alpha_{2}}, S_{\alpha_{1}}\right)-\operatorname{Cov}\left(N_{\alpha_{2}}, Q_{0}-Q_{x}\right) \\
& =\operatorname{Cov}\left(N_{\alpha_{2}}, Q_{0}\right)-\operatorname{Cov}\left(N_{\alpha_{2}}, Q_{x}\right)
\end{aligned}
$$

pois, $N_{\alpha_{2}}$ é função determinística de $S_{\alpha_{2}}(-\infty, x)$ e $P$ que são ambos independentes de $S_{\alpha_{1}}$.

Para calcular $\operatorname{Cov}\left(N_{\alpha_{2}}, Q_{0}\right)$ primeiro veja que o processo multilinha é reversível no tempo. Então utilizamos um argumento análogo ao usado para calcular $\operatorname{Cov}\left(N_{\rho}, S_{\lambda}\right)$ acima só que com $N_{\alpha_{2}}$ fazendo o papel de $S_{\lambda}$ e $Q_{0}$ no lugar de $N_{\rho}$, isto é alteramos o processo $N_{\alpha_{2}}$ e fazemos o tempo voltar de $t$ para zero, observando como essa alteração afeta o valor de $Q_{0}$. Para que a partícula $Z_{y}(0)$ acrescida à $N_{\alpha_{2}}$ no ponto $y$ modifique o número de pessoas na fila na origem $Q_{0}$ a fila deve estar ocupada $\left(Q_{0}>0\right)$ e $Z_{y}(0)$ deve pertencer ao intervalo $(0, Y)$ onde Y é o início do período de ocupação ao qual $Q_{0}$ pertence. Observando que 
$Y$ não depende de $t$ (de fato, $Y=O(1)$ ), temos que

$$
\begin{aligned}
\operatorname{Cov}\left(N_{\alpha_{2}}, Q_{0}\right) & =\left.\lambda \partial \gamma\right|_{\gamma=0} \mathbb{E}\left(Q_{0}^{\gamma}\right) \\
& =\lambda \rho \int_{0}^{x} \mathbb{P}\left(Z_{y}(0) \geq 0, Z_{y}(0) \leq Y\right) d y \\
& =\lambda \rho \int_{0}^{x} \mathbb{P}\left(Z^{\nu_{\lambda}}(t) \leq x-y, Z^{\nu_{\lambda}}(t) \geq x-Y-y\right) d y \\
& =\lambda \rho \int_{0}^{x} \mathbb{P}\left(x-Z^{\nu_{\lambda}}(t)>y\right)-\mathbb{P}\left(x-Z^{\nu_{\lambda}}(t) \geq y+O(1)\right) d y \\
& =O(1)
\end{aligned}
$$

Agora vamos estudar $\operatorname{Cov}\left(N_{\alpha_{2}}, Q_{x}\right)$. Novamente acrescentamos uma partícula $Z_{y}(t)$ a $N_{\alpha_{2}}$ no ponto $y<x$. Temos que $Z_{y}(0)<x$ e como $Q_{x}$ é determinado por $S_{\alpha_{2}}(x,+\infty)$ e $S_{\alpha_{1}}(x,+\infty)$ então $Z_{y}(0)$ não afeta $Q_{x}$. Assim $\operatorname{Cov}\left(N_{\alpha_{2}}, Q_{x}\right)=0$.

Logo

$$
\operatorname{Cov}\left(N_{\xi}, S_{\xi}\right)=\lambda \mathbb{E}\left(x-Z^{\nu_{\lambda}}(t)\right)_{+}+\rho \mathbb{E}\left(x-Z^{\nu_{\rho}}(t)\right)_{+}-O(1)
$$

Resta calcular a covariância entre $S_{\xi}$ e $W_{\xi}$. Temos:

$$
\begin{aligned}
\operatorname{Cov}\left(S_{\xi}, W_{\xi}\right) & =\operatorname{Cov}\left(S_{\lambda}-S_{\rho}, W_{\lambda}-W_{\rho}\right) \\
& =\operatorname{Cov}\left(S_{\lambda}, W_{\lambda}\right)+\operatorname{Cov}\left(S_{\rho}, W_{\rho}\right)-\operatorname{Cov}\left(S_{\lambda}, W_{\rho}\right)-\operatorname{Cov}\left(S_{\rho}, W_{\lambda}\right) \\
& =-\operatorname{Cov}\left(S_{\lambda}, W_{\rho}\right)-\operatorname{Cov}\left(S_{\rho}, W_{\lambda}\right) \\
& =-\operatorname{Cov}\left(S_{\lambda}, W_{\rho}\right)
\end{aligned}
$$

onde a última igualdade segue do fato que $W_{\lambda}$ é função determinística de $P$ e $S_{\alpha_{2}}(-\infty, 0)$ enquanto $S_{\rho}$ é função determinística de $S_{\alpha_{2}}(0,+\infty)$ e $S_{\alpha_{1}}(0,+\infty)$ sendo estes independentes dos anteriores.

Por fim, a $\operatorname{Cov}\left(S_{\lambda}, W_{\rho}\right)$. Mais uma vez acrescentamos uma partícula $Z_{y}$ a $S_{\lambda}$ no ponto $y$. Como antes, se $Q_{y}=0$ então $S_{\rho}=S_{\rho}^{\gamma}$ o que implica $W_{\rho}=W_{\rho}^{\gamma}$. Suponha $Q_{y}>0$. Isso gera duas discrepâncias entre $S_{\rho}$ e $S_{\rho}^{\gamma}, Z_{y}$ e $\bar{Z}_{\bar{y}}$, com $\bar{y}=y-O(1)$. Se $\bar{y}>0$, então $W_{\rho}=W_{\rho}^{\gamma}$. Agora, se $\bar{y} \leq 0$ então $y$ pertence 
ao mesmo período de ocupação da origem, ie, $y \in(0, Y)$. Neste caso teríamos $W_{\rho}^{\gamma}-W_{\rho}=\mathbf{1}\left[\bar{Z}_{\bar{y}}(t)>0\right]$. Em resumo,

$$
\begin{aligned}
\left|\operatorname{Cov}\left(S_{\lambda}, W_{\rho}\right)\right| & =\lambda \lim _{\gamma \rightarrow 0} \frac{\left|\mathbb{E}\left(W_{\rho}^{\gamma}-W_{\rho}\right)\right|}{\gamma} \\
& =\lambda \mathbb{P}\left(Q_{y}>0\right) \mathbb{E}\left(\int_{0}^{x} \mathbf{1}_{\left[\bar{Z}_{\bar{y}}(t)>0\right]} \mathbf{1}_{[y \leq Y]} d y\right) \\
& \leq \lambda \rho \mathbb{E}(Y) \\
& =O(1)
\end{aligned}
$$

Assim

$$
\operatorname{Cov}\left(S_{\xi}, W_{\xi}\right)=O(1)
$$

Como a aplicação $(x, t) \mapsto\left(\gamma x, \frac{t}{\gamma}\right)$ transforma um processo de Hammersley em equilíbrio de taxa $\gamma$ em um Hammersley de taxa 1, temos que $\gamma Z^{\nu_{\gamma}}(t)=$ $Z^{\nu_{1}}(t / \gamma)$ em lei. Da onde obtemos que

$$
\begin{aligned}
\gamma \mathbb{E} & \left(x-Z_{\gamma}(t)\right)_{+}=\mathbb{E}\left(\gamma x-Z^{\nu_{1}}(t / \gamma)\right)_{+} \\
& =\mathbb{E}\left(\left[\gamma x-Z^{\nu_{1}}(t / \gamma)\right] \mathbf{1}_{\left[Z^{\nu_{1}}(t / \gamma) \leq \gamma x\right]}\right) \\
& =\mathbb{E}\left([\gamma x-t / \gamma] \mathbf{1}_{\left[Z^{\nu_{1}}(t / \gamma) \leq \gamma x\right]}\right)+\mathbb{E}\left(\left[t / \gamma-Z^{\nu_{1}}(t / \gamma)\right] \mathbf{1}_{\left[Z^{\nu_{1}}(t / \gamma) \leq \gamma x\right]}\right) \\
& =(\gamma x-t / \gamma) \mathbb{P}\left(Z^{\nu_{1}}(t / \gamma) \leq \gamma x\right)+\mathbb{E}\left(\left[t / \gamma-Z^{\nu_{1}}(t / \gamma)\right] \mathbf{1}_{\left[Z^{\nu_{1}}(t / \gamma) \leq \gamma x\right]}\right)
\end{aligned}
$$

Logo, por (6.4), (6.5),(6.6) e (6.2)

$$
\begin{aligned}
\operatorname{Cov}( & \left.N_{\xi}, W_{\xi}\right)=\operatorname{Cov}\left(N_{\xi}, S_{\xi}\right)+\operatorname{Cov}\left(S_{\xi}, W_{\xi}\right)-\operatorname{Var}\left(N_{\xi}\right) \\
= & \lambda \mathbb{E}\left(x-Z^{\nu_{\lambda}}(t)\right)_{+}+\rho \mathbb{E}\left(x-Z^{\nu_{\rho}}(t)\right)_{+}-(\lambda+\rho) x+O(1) \\
= & (\lambda x-t / \lambda) \mathbb{P}\left(Z^{\nu_{1}}(t / \lambda) \leq \lambda x\right)+(\rho x-t / \rho) \mathbb{P}\left(Z^{\nu_{1}}(t / \rho) \leq \rho x\right) \\
& +\mathbb{E}\left(\left[t / \lambda-Z^{\nu_{1}}(t / \lambda)\right] \mathbf{1}_{\left[Z^{\nu_{1}}(t / \lambda) \leq \lambda x\right]}\right)+\mathbb{E}\left(\left[t / \rho-Z^{\nu_{1}}(t / \rho)\right] \mathbf{1}_{\left[Z^{\nu_{1}}(t / \rho) \leq \rho x\right]}\right) \\
& -(\lambda+\rho) x+O(1)
\end{aligned}
$$


Finalmente, por (6.2), (6.3) e (6.7)

$$
\begin{aligned}
\operatorname{Var} L_{\xi}(x, & t)=\operatorname{Var}\left(N_{\xi}\right)+\operatorname{Var}\left(W_{\xi}\right)+2 \operatorname{Cov}\left(N_{\xi}, W_{\xi}\right) \\
=(\lambda & +\rho) x+\left(\frac{1}{\lambda}+\frac{1}{\rho}\right) t \\
& +2 \mathbb{E}\left(\lambda x-Z^{\nu_{1}}(t / \lambda)\right)_{+}+2 \mathbb{E}\left(\rho x-Z^{\nu_{1}}(t / \rho)\right)_{+}-2(\lambda+\rho) x \\
& +O(1) \\
=(\lambda & +\rho)\left(\frac{t}{\lambda \rho}-x\right) \\
+ & 2(\lambda x-t / \lambda) \mathbb{P}\left(Z^{\nu_{1}}(t / \lambda) \leq \lambda x\right)+2(\rho x-t / \rho) \mathbb{P}\left(Z^{\nu_{1}}(t / \rho) \leq \rho x\right) \\
+ & 2 \mathbb{E}\left(\left[t / \lambda-Z^{\nu_{1}}(t / \lambda)\right] \mathbf{1}_{\left[Z^{\nu_{1}}(t / \lambda) \leq \lambda x\right]}\right)+2 \mathbb{E}\left(\left[t / \rho-Z^{\nu_{1}}(t / \rho)\right] \mathbf{1}_{\left[Z^{\nu_{1}}(t / \rho) \leq \rho x\right]}\right) \\
+ & O(1)
\end{aligned}
$$

Teorema 6.2. Seja $L_{\xi}(x, t)$ o fluxo de partículas de segunda classe da origem $a(x, t)$ em um processo de duas classes com medida inicial invariante. Seja $V>0$ uma constante e $x=x_{t}$ uma função de $t$. Se $\lim _{t \rightarrow+\infty} \frac{x_{t}}{t}=V$ então

$$
\operatorname{Var} L_{\xi}\left(x_{t}, t\right)=f(t)+g(t)+O(1)
$$

onde

$$
\begin{aligned}
f(t)= & f(V, t) \\
& :=(\lambda+\rho)\left(\frac{t}{\lambda \rho}-x_{t}\right)+ \\
& \quad+2\left(\lambda x_{t}-t / \lambda\right) \mathbb{P}\left(Z^{\nu_{1}}(t / \lambda) \leq \lambda x_{t}\right)+2\left(\rho x_{t}-t / \rho\right) \mathbb{P}\left(Z^{\nu_{1}}(t / \rho) \leq \rho x_{t}\right)
\end{aligned}
$$

é da ordem de $t$

$$
\begin{aligned}
g(t) & =g(V, t) \\
& :=2 \mathbb{E}\left(\left[t / \lambda-Z^{\nu_{1}}(t / \lambda)\right] \mathbf{1}_{\left[Z^{\nu_{1}}(t / \lambda) \leq \lambda x_{t}\right]}\right)+2 \mathbb{E}\left(\left[t / \rho-Z^{\nu_{1}}(t / \rho)\right] \mathbf{1}_{\left[Z^{\nu_{1}}(t / \rho) \leq \rho x_{t}\right]}\right)
\end{aligned}
$$

é da ordem de $t^{2 / 3}$. 
Em particular,

$$
\lim _{t \rightarrow \infty} \frac{\operatorname{Var} L_{\xi}(V t, t)}{t}=\lim _{t \rightarrow \infty} \frac{f(t)}{t}= \begin{cases}(\lambda+\rho)\left(\frac{1}{\lambda \rho}-V\right) & \text { se } V \leq \frac{1}{\lambda^{2}} \\ (\lambda-\rho)\left(\frac{1}{\lambda \rho}+V\right) & \text { se } V \in\left(\frac{1}{\lambda^{2}}, \frac{1}{\rho^{2}}\right) \\ (\lambda+\rho)\left(V-\frac{1}{\lambda \rho}\right) & \text { se } V \geq \frac{1}{\rho^{2}}\end{cases}
$$

\section{Prova do Teorema 6.2.}

A equação $\operatorname{Var} L_{\xi}(x, t)=f(V, t)+g(V, t)+O(1)$ segue diretamente da proposição 6.2 .

O limite de $\frac{f(V, t)}{t}$ segue da definição da $f$ e da lei dos grandes números para $Z^{\nu_{1}}$, ie, com probabilidade $1, \lim _{t \rightarrow \infty} \frac{Z^{\nu_{1}}(t)}{t}=1$.

Quanto a $g(V, t)$ temos que, pela proposição 6.1:

$$
\begin{aligned}
0 & =2 \mathbb{E}\left(t / \lambda-Z^{\nu_{1}}(t / \lambda)\right)+2 \mathbb{E}\left(t / \rho-Z^{\nu_{1}}(t / \rho)\right) \\
& <g(t) \\
& \leq 2 \mathbb{E}\left(t / \lambda-Z^{\nu_{1}}(t / \lambda)\right)_{+}+2 \mathbb{E}\left(t / \rho-Z^{\nu_{1}}(t / \rho)\right)_{+}
\end{aligned}
$$

Sabemos que as duas últimas esperanças são da ordem de $t^{2 / 3}$ (ver por exemplo Cator e Groeneboom [9]). Como $g(V, t)$ é continua, segue que $g(V, t)$ é da ordem de $t^{2 / 3}$. 


\section{Referências Bibliográficas}

[1] D. Aldous and P. Diaconis, Hammersley's interacting particle process and longest increasing subsequences, Probab. Theory Related Fields 103 (1995), no. 2, 199-213. MR1355056 (96k:60017) 5, 7

[2] J. Baik and E. M. Rains, Limiting distributions for a polynuclear growth model with external sources, J. Statist. Phys. 100 (2000), no. 3-4, 523-541. MR1788477 (2001h:82067) 1, 2

[3] J. Baik, P. Deift and K. Johansson, On the distribution of the length of the longest increasing subsequence of random permutations, J. Amer. Math. Soc. 12 (1999), no. 4, 1119-1178. MR1682248 (2000e:05006) 2

[4] M. Balázs, Growth fluctuations in a class of deposition models, Ann. Inst. H. Poincaré Probab. Statist. 39 (2003), no. 4, 639-685. MR1983174 (2005j:60178a) 1

[5] M. Balázs, E. Cator and T. Seppäläinen, Cube root fluctuations for the corner growth model associated to the exclusion process, Electron. J. Probab. 11 (2006), no. 42, 1094-1132 (electronic). MR2268539 (2007k:60312) 2

[6] M. Balázs and T. Seppäläinen, Order of current variance and diffusivity in the asymmetric simple exclusion process, Ann. of Math. (2) 171 (2010), no. 2, 1237-1265. MR2630064 (2011m:60287) 2

[7] G. Ben Arous and I. Corwin, Current fluctuations for TASEP: a proof of the Prähofer-Spohn conjecture, Ann. Probab. 39 (2011), no. 1, 104-138. MR2778798 (2012e:60249) 2

[8] E. Cator and P. Groeneboom, Hammersley's process with sources and sinks, Ann. Probab. 33 (2005), no. 3, 879-903. MR2135307 (2006b:60216) 1, 8, 17 
[9] E. Cator and P. Groeneboom, Second class particles and cube root asymptotics for Hammersley's process, Ann. Probab. 34 (2006), no. 4, 1273-1295. MR2257647 (2008e:60021) 1, 2, 3, 4, 8, 22, 25, 26, 49, 50, 56, 62

[10] E. Cator and L.P.R. Pimentel, Busemman functions and equilibrium measures in last-passage percolation models., To appear in Probab. Theory Related Fields. (2011), Available from PTRF Online First. 3, 9

[11] E. A. Cator, L. P. R. Pimentel and M. W. A. Souza, Influence of the initial condition in equilibrium last-passage percolation models, Electron. Commun. Probab. 17 (2012), no. 7, 7 pp. MR2878742 25

[12] C. Coletti, P. A. Ferrari and L.P.R. Pimentel, The variance of the shock in the HAD process, Preprint (2008), math.PR/0801.2526 2008 42, 44

[13] P. A. Ferrari, Shocks in the Burgers equation and the asymmetric simple exclusion process, in Statistical physics, automata networks and dynamical systems (Santiago, 1990), 25-64, Math. Appl., 75 Kluwer Acad. Publ., Dordrecht. MR1263704 2

[14] P. A. Ferrari and L. R. G. Fontes, Shock fluctuations in the asymmetric simple exclusion process, Probab. Theory Related Fields 99 (1994), no. 2, 305-319. MR1278887 (95h:60159) 2, 3, 11, 42

[15] P. A. Ferrari and L. R. G. Fontes, Current fluctuations for the asymmetric simple exclusion process, Ann. Probab. 22 (1994), no. 2, 820-832. MR1288133 (95j:60162) 1, 11

[16] P. A. Ferrari and J. B. Martin, Multiclass Hammersley-Aldous-Diaconis process and multiclass-customer queues, Ann. Inst. Henri Poincaré Probab. Stat. 45 (2009), no. 1, 250-265. MR2500238 (2010c:60288) 3, 9, 18, 20, 24

[17] P. A. Ferrari and J. B. Martin, Stationary distributions of multi-type totally asymmetric exclusion processes, Ann. Probab. 35 (2007), no. 3, 807-832. MR2319708 (2008h:60403) 3

[18] P. L. Ferrari and H. Spohn, Scaling limit for the space-time covariance of the stationary totally asymmetric simple exclusion process, Comm. Math. Phys. 265 (2006), no. 1, 1-44. MR2217295 (2007g:82038a) 2

[19] P. Groeneboom, Ulam's problem and Hammersley's process, Ann. Probab. 29 (2001), no. 2, 683-690. MR1849174 (2002f:60016) 1 
[20] J. M. Hammersley, A few seedlings of research, in Proceedings of the Sixth Berkeley Symposium on Mathematical Statistics and Probability (Univ. California, Berkeley, Calif., 1970/1971), Vol. I: Theory of statistics, 345-394, Univ. California Press, Berkeley, CA. MR0405665 (53 \#9457) 1

[21] K. Johansson, Transversal fluctuations for increasing subsequences on the plane, Probab. Theory Related Fields 116 (2000), no. 4, 445-456. MR1757595 (2001e:60210) 2

[22] T. Seppäläinen, A microscopic model for the Burgers equation and longest increasing subsequences, Electron. J. Probab. 1 (1996), no. 5, approx. 51 pp. (electronic). MR1386297 (97d:60162) 1, 2

[23] T. Seppäläinen, Diffusive fluctuations for one-dimensional totally asymmetric interacting random dynamics, Comm. Math. Phys. 229 (2002), no. 1, 141-182. MR1917677 (2003g:82069) 2

[24] T. Seppäläinen, Perturbation of the equilibrium for a totally asymmetric stick process in one dimension, Ann. Probab. 29 (2001), no. 1, 176-204. MR1825147 (2002c:60173) 27 